\title{
The Milky Way has no in-situ halo other than the heated thick disc
}

\section{Composition of the stellar halo and age-dating the last significant merger with Gaia DR2 and APOGEE}

\author{
P. Di Matteo ${ }^{1,2}$, M. Haywood ${ }^{1,2}$, M. D. Lehnert ${ }^{2}$, D. Katz ${ }^{1}$, S. Khoperskov ${ }^{3,4}$, O. N. Snaith ${ }^{1,5}$, \\ A. Gómez ${ }^{1}$, and N. Robichon ${ }^{1}$ \\ 1 GEPI, Observatoire de Paris, PSL Research University, CNRS, Place Jules Janssen, 92190 Meudon, France \\ e-mail: paola.dimatteo@obspm. fr \\ 2 Sorbonne Université, CNRS UMR 7095, Institut d'Astrophysique de Paris, 98bis bd Arago, 75014 Paris, France \\ 3 Max Planck Institute for Extraterrestrial Physics, 85741 Garching, Germany \\ 4 Institute of Astronomy, Russian Academy of Sciences, Pyatnitskaya st., 48, 119017 Moscow, Russia \\ 5 School of Physics, Korea Institute for Advanced Study, 85 Hoegiro, Dongdaemun-gu, Seoul 02455, Republic of Korea
}

Received 19 December 2018 / Accepted 9 September 2019

\begin{abstract}
Previous studies based on the analysis of Gaia DR2 data have revealed that accreted stars, possibly originating from a single progenitor satellite, are a significant component of the halo of our Galaxy, potentially constituting most of the halo stars at $[\mathrm{Fe} / \mathrm{H}]<-1$ within a few kpc from the Sun and beyond. In this paper, we couple astrometric data from Gaia DR2 with elemental abundances from APOGEE DR14 to characterise the kinematics and chemistry of in-situ and accreted populations up to $[\mathrm{Fe} / \mathrm{H}] \sim-2$. Accreted stars appear to significantly impact the galactic chemo-kinematic relations, not only at $[\mathrm{Fe} / \mathrm{H}]<-1$, but also at metallicities typical of the thick and metal-poor thin discs. They constitute about $60 \%$ of all stars at $[\mathrm{Fe} / \mathrm{H}]<-1$, the remaining $40 \%$ being made of (metal-weak) thick-disc stars. We find that the stellar kinematic fossil record shows the imprint left by this accretion event, which heated the old galactic disc. We are able to age-date this kinematic imprint, showing that the accretion occurred between nine and $11 \mathrm{Gyr}$ ago, and that it led to the last significant heating of the galactic disc. An important fraction of stars with abundances typical of the (metal-rich) thick disc, and heated by this interaction, is now found in the galactic halo. Indeed, about half of the kinematically defined halo at few kpc from the Sun is composed of metal-rich thick-disc stars. Moreover, we suggest that this metal-rich thick-disc component dominates the stellar halo of the inner Galaxy. The new picture that emerges from this study is one where the standard, non-rotating in-situ halo population, the collapsed halo, seems to be more elusive than ever.
\end{abstract}

Key words. Galaxy: abundances - Galaxy: stellar content - Galaxy: kinematics and dynamics - Galaxy: structure - Galaxy: evolution

\section{Introduction}

The presence of accreted stars in galaxy halos is a natural expectation of $\Lambda$ CDM Cosmology (Cole 1991; White \& Frenk 1991). Stars and stellar systems deposited in our galactic halo by past accretion events have been known and postulated for decades (Searle \& Zinn 1978; Zinn et al. 1993, 1996; Majewski et al. 1996; Helmi et al. 1999; Chiba \& Beers 2000; Venn et al. 2004; Bullock et al. 2004; Font et al. 2006; Carollo et al. 2007; Bell et al. 2008; De Lucia \& Helmi 2008; Johnston et al. 2008; Forbes \& Bridges 2010; Xue et al. 2011; Leaman et al. 2013; Pillepich et al. 2015). Chemical abundances of stars in the solar vicinity and on a larger scale of a few kpc have provided evidence that two halo components co-exist: an $\alpha$-enhanced, metal-poor population, which possibly set the initial conditions for the formation of the galactic disc, and a low $\alpha$-abundance, metal-poor population, possibly accreted early in the evolution of our Galaxy (Nissen \& Schuster 2010, 2011; Navarro et al. 2011; Ramírez et al. 2012; Schuster et al. 2012; Hawkins et al. 2015; Hayes et al. 2018).

Together with the discovery of a number of thin stellar streams, possibly associated with disrupted globular clusters (Ibata et al. 2018; Malhan et al. 2018; Price-Whelan \& Bonaca 2018), the analysis of the first and second Gaia releases (Gaia Collaboration et al. 2016, 2017, 2018a) have revealed the presence of tidal debris from an ancient massive accretion. (Belokurov et al. 2018; Haywood et al. 2018; Myeong et al. 2018; Helmi et al. 2018; Mackereth et al. 2019; Fattahi et al. 2019). As shown by Haywood et al. (2018), and later confirmed by Helmi et al. (2018), stars belonging to this massive accretion event mostly redistribute along the bluer of the two sequences discovered in the Gaia DR2 HR diagram of kinematically selected halo stars (Gaia Collaboration et al. 2018b). Their chemical abundances overlap with the low- $\alpha$ sequence discovered by Nissen \& Schuster (2010, see Haywood et al. 2018) and extensively studied by Hayes et al. (2018, see Haywood et al. 2018; Helmi et al. 2018). The dynamics and orbits of these low- $\alpha$ stars, e.g., their positions in a "Toomre diagram", are such that they dominate regions with no or retrograde rotation and high total orbital energy (Koppelman et al. 2018; Haywood et al. 2018). This is true even if the overlap with the red HR diagram sequence in Gaia DR2 is significant (Haywood et al. 2018), as expected in a scenario where the stellar halo is made of both accreted and in-situ disc stars ${ }^{1}$ (Jean-Baptiste et al. 2017).

1 By "in-situ disc stars", we mean stars that formed in the early Milky Way disc and were then heated by interactions. (Purcell et al. 2010; Zolotov et al. 2010; Font et al. 2011; Qu et al. 2011a; McCarthy et al. 2012) 
Accreted stars have been suggested to be the dominant metalpoor (i.e. $[\mathrm{Fe} / \mathrm{H}]<-1)$ halo component at few kpc from the Sun and beyond (Haywood et al. 2018; Belokurov et al. 2018; Iorio \& Belokurov 2019) and may extend to relatively high metallicities (i.e. at $[\mathrm{Fe} / \mathrm{H}]>-1$; see Nissen \& Schuster 2010; Hayes et al. 2018).

In this paper, by coupling kinematics and chemical abundances, we aim to discuss the imprint of the accreted stars on the galactic chemo-kinematic relations, at metallicities typical of the halo, as well as the metal-poor thin and thick discs. Secondly, we aim to estimate each component's relative fraction in different regions of the $[\mathrm{Fe} / \mathrm{H}]-[\mathrm{Mg} / \mathrm{Fe}]$ plane, especially at $[\mathrm{Fe} / \mathrm{H}]<-1$, where a discrimination on the basis of the chemical abundances alone is likely not possible. Thirdly, we intend to age-date the last significant accretion event experienced by the Galaxy by making use of kinematics to investigate signatures of the heating of the early galactic disc, and by using the abundances in the early galactic disc to constrain the time when this heating event occurred. Finally, the accretion of relatively massive satellites is expected to generate a significant fraction of stars with halo kinematics, but abundances typical of the early disc from which they were kicked out. Stars heated by merging events should have kinematics which overlap with the accreted population (Jean-Baptiste et al. 2017). We conclude our analysis by investigating the presence of in-situ stars with disc chemistry, but with halo kinematics to estimate their relative fractions of their original population, and of the total halo population within few kpc from the Sun.

The paper is organised as follows: in Sect. 2, we state how we selected the sample of stars used in this analysis. In Sect. 3, we present our results, which include the mean galactic chemokinematic relations (Sect. 3.1), the relative fractions of in-situ and accreted stars in the $[\mathrm{Fe} / \mathrm{H}]-[\mathrm{Mg} / \mathrm{Fe}]$ plane (Sect. 3.2), and how we age-dated the last significant accretion event experienced by our Galaxy (Sect. 3.3). In Sect. 4, we discuss the implications of our findings, and finally, in Sect. 5, we summarise our conclusions.

\section{Data}

The sample that we analyse in this paper is the result of crossmatching the Gaia DR2 (Gaia Collaboration et al. 2018a) with APOGEE data from DR14 (Majewski et al. 2017), using the CDS X-match service ${ }^{2}$. To construct this sample, we selected stars from the two catalogues with a position mismatch tolerance of $0.5 \mathrm{arcsec}$, and retained only those with positive parallaxes $\pi$, relative error on parallaxes $\sigma_{\pi} / \pi<0.2$, and a signal-to-noise ratio on the APOGEE spectra, $S / N>100$. All line-of-sight velocities used in this paper are from APOGEE. Following the study of Fernández-Alvar et al. (2019a), we applied additional selection criteria only retaining stars with effective temperatures, $T_{\text {eff }}>4000$, and gravities, $1<\log (g)<3.5$. Finally, we also removed all APOGEE stars with ASCAPFLAG and STARFLAG warning of any problems with the determinations of the atmospheric parameters (specifically those with a warning about the reliability of the effective temperature, $\log (g)$, rotation, and having a very bright neighbour). After applying all these selection criteria, our final sample consists of 61789 stars, whose density distribution, projected onto the galactic and meridional planes, is shown in Fig. 1. As expected, most of the stars in the analysed sample are at a distance of $2-3 \mathrm{kpc}$ from the Sun, with a dearth of stars in the forth quadrant, due to the lack of coverage

\footnotetext{
2 http://cdsxmatch.u-strasbg.fr/xmatch
}
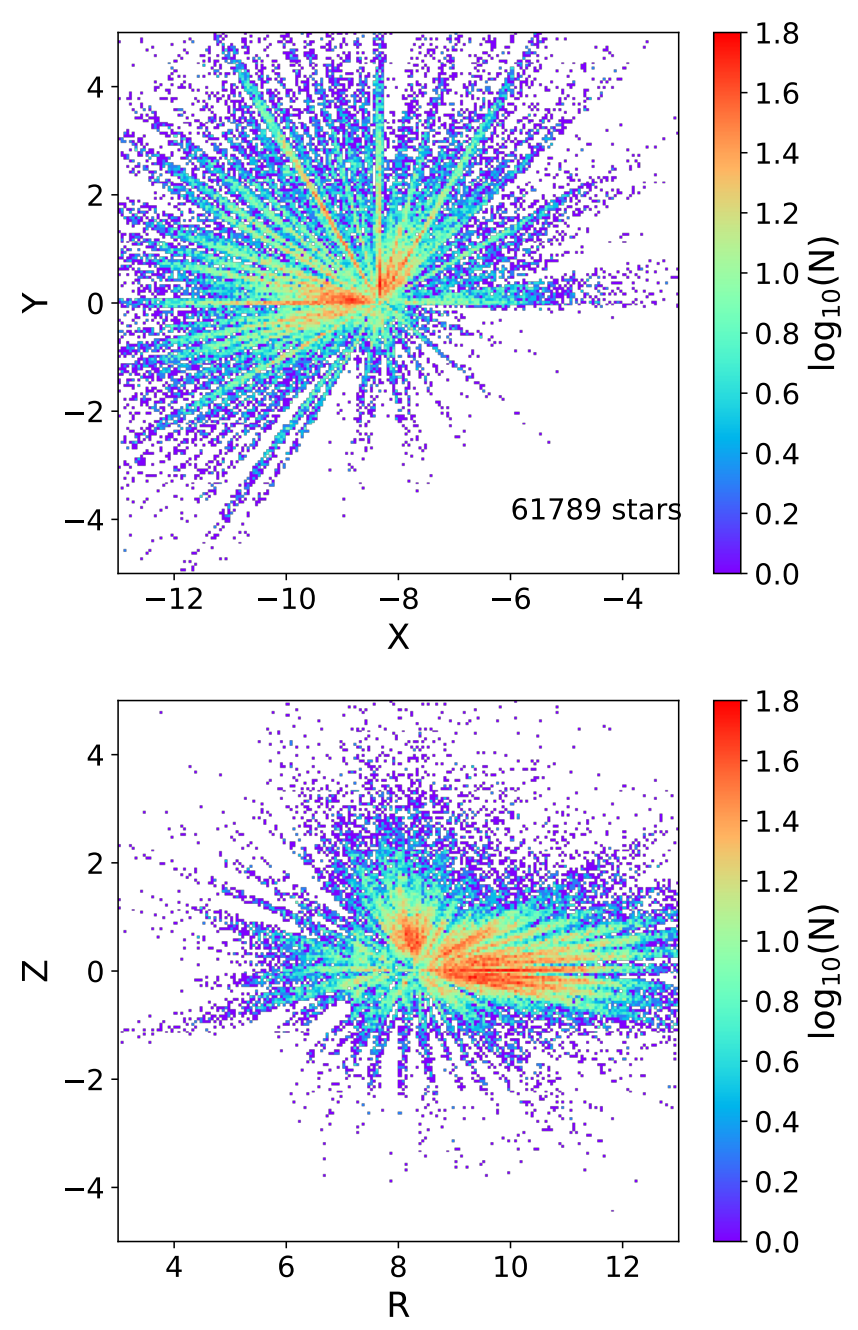

Fig. 1. Spatial distribution of stars in the sample we are analysing in $x-y$ (top panel) and $R-z$ (bottom panel) planes. Total number of stars in the sample is provided in lower left-hand corner of top panel. In both maps, number of stars, in logarithmic scale, per pixel, the pixel size being $50 \times 50 \mathrm{pc}^{2}$, is colour-coded as given in the bar on the right-hand side of each panel. The Sun lies at $x=-8.34 \mathrm{kpc}, y=0$ and $z=27 \mathrm{pc}$.

of this area in the APOGEE footprint. For calculating positions and velocities in the galactocentric rest-frame, we assumed an inplane distance of the Sun from the galactic centre, $R_{\odot}=8.34 \mathrm{kpc}$ (Reid et al. 2014), a height of the Sun above the galactic plane, $z_{\odot}=27 \mathrm{pc}$ (Chen et al. 2001), a velocity for the Local Standard of Rest, $V_{\mathrm{LSR}}=240 \mathrm{~km} \mathrm{~s}^{-1}$ (Reid et al. 2014), and a peculiar velocity of the Sun with respect to the LSR, $U_{\odot}=11.1 \mathrm{~km} \mathrm{~s}^{-1}$, $V_{\odot}=12.24 \mathrm{~km} \mathrm{~s}^{-1}, W_{\odot}=7.25 \mathrm{~km} \mathrm{~s}^{-1}$ (Schönrich et al. 2010). Individual uncertainties in the velocities of stars in the sample, due to the propagation of the uncertainties on the observables (parallaxes, proper motions and radial velocities) are discussed in Appendix A. Parallaxes were corrected by the zero-point offset of -0.03 mas (Arenou et al. 2018; Gaia Collaboration et al. 2018a; Lindegren et al. 2018), and distances were derived by inverting parallaxes. While the correction of the zero-point offset affects, as expected, the absolute values of our derived relations, and mean/median kinematics, all trends and conclusions presented in the following section of the paper are also maintained when no correction of the parallax zero-point is made.

In the following sections of this paper, we discuss fractions of in-situ (thick-disc) stars relative to accreted stars. The APOGEE selection function is not expected to introduce any 

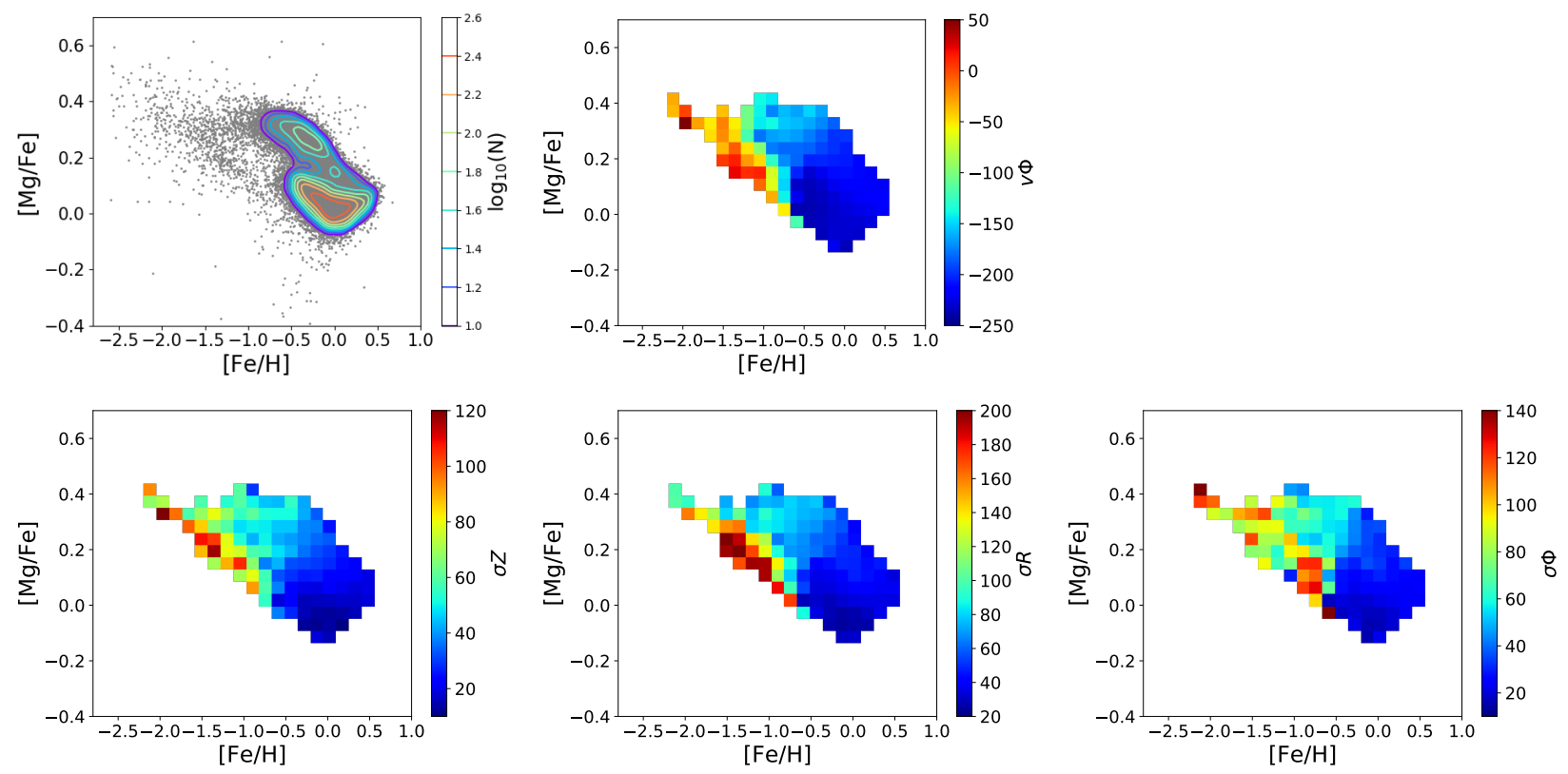

Fig. 2. Top-left panel: distribution of stars in our sample in $[\mathrm{Fe} / \mathrm{H}]-[\mathrm{Mg} / \mathrm{Fe}]$ plane. Coloured lines correspond to isodensity contours, in logarithmic scale, as indicated in the colour bar on the right-hand side of the panel. Top-right panel: mean azimuthal velocity of stars in $[\mathrm{Fe} / \mathrm{H}]-[\mathrm{Mg} / \mathrm{Fe}]$ plane. In this and all subsequent panels, the pixel size is $0.152 \times 0.044$, and only pixels containing more than ten stars are shown. Bottom-left panel: mean vertical velocity dispersions of stars in $[\mathrm{Fe} / \mathrm{H}]-[\mathrm{Mg} / \mathrm{Fe}]$ plane. Bottom-middle panel: mean radial velocity dispersions of stars in $[\mathrm{Fe} / \mathrm{H}]-[\mathrm{Mg} / \mathrm{Fe}]$ plane. Bottom-right panel: mean azimuthal velocity dispersions of stars in $[\mathrm{Fe} / \mathrm{H}]-[\mathrm{Mg} / \mathrm{Fe}]$ plane.

bias against any specific population at a given metallicity, $\alpha$-abundance, or kinematics, because the selection criteria in colour and magnitude of the survey (see Majewski et al. 2017) are not related to these parameters. The colour cut of the survey introduces a selection of metal-poor stars, but there is no reason that this would introduce a bias against a specific population, in the metallicity range studied in this paper. So we do expect the fractions discussed here to be representative of a local sample of the Galaxy. However, it goes without saying that the relative fractions of the different populations are affected by the distance limit of the APOGEE sample, and may not be representative of the entire Milky Way. Figure 1 shows the complex distribution of the APOGEE stars resulting from the adopted footprint of the survey. It is seen in particular that the APOGEE footprint favours anticentre directions, whereas the thick-disc population is known to be less well-represented, because of its short scale length. It has recently been shown that the radial distributions of these two populations are widely different (Sahlholdt et al. 2019), the accreted stars having a much more uniform distribution than thick-disc stars within a few kpc of the Sun. It is therefore possible that these fractions are biased in the sense that the thick disc is relatively less represented locally.

In Fig. 2, we show the distribution of our sample in the $[\mathrm{Mg} / \mathrm{Fe}]-[\mathrm{Fe} / \mathrm{H}]$ plane. While the vast majority of the stars have $[\mathrm{Fe} / \mathrm{H}] \geq-1$, and occupy the two chemically-defined sequences of the galactic thick and thin discs, about $1.5 \%$ of the sample has $[\mathrm{Fe} / \mathrm{H}] \leq-1$. These relatively low $\mathrm{Fe}$ abundance stars lie along two sequences: a high $[\mathrm{Mg} / \mathrm{Fe}]$-abundance sequence, which joins to the thick-disc sequence at high metallicities, but with a possible dip in the density of stars at $[\mathrm{Fe} / \mathrm{H}] \sim-1$ (Hayes et al. 2018; Gaia Collaboration et al. 2018b), and a sequence extending from high $[\mathrm{Mg} / \mathrm{Fe}]$ abundances, at metallicities $[\mathrm{Fe} / \mathrm{H}] \sim-2$, to $[\mathrm{Mg} / \mathrm{Fe}]$ abundances about 0.2 lower, at $[\mathrm{Fe} / \mathrm{H}] \sim-1$. The continuation of the two sequences, at low $([\mathrm{Fe} / \mathrm{H}] \sim-2)$ and high $([\mathrm{Fe} / \mathrm{H}] \sim-1)$ metallicities is still uncertain and difficult to constrain at present. However, we subsequently show that, by coupling chemical abundances with kinematics, it is possible to relate the low- $[\mathrm{Mg} / \mathrm{Fe}]$ sequence to the metal-poor tail of the thin disc, namely, thin-disc stars with $[\mathrm{Fe} / \mathrm{H}] \sim-0.5$ and solar and sub-solar $[\mathrm{Mg} / \mathrm{Fe}]$.

\section{Results}

Before presenting the results, we wish to introduce the nomenclature and conventions adopted in this paper, as this often leads to confusion in this type of study. The definition and the borders of the galactic stellar populations are not always trivial to set, because of the overlap that all populations (from those of the bulge to the halo) passing through the thin and thick discs show.

The galactic halo: In this paper, the halo is sometimes defined on the basis of its chemistry, that is as stars with $[\mathrm{Fe} / \mathrm{H}]<-1$. Sometimes we define the halo kinematically, that is, made of stars with absolute velocities, relative to the LSR, greater than $180 \mathrm{~km} \mathrm{~s}^{-1}$. We endeavour to be specific regarding which definition we employ and, in Sect. 4, we summarise all our findings in terms of what the "galactic halo" consists of within a few kpc of the Sun.

Convention adopted for the azimuthal velocities: In our choice of the Galactocentric coordinate system, the Sun lies on the $\mathrm{x}$-axis with a negative value of $x=-8.34 \mathrm{kpc}$, and the $V_{\odot}$ is positive, which is parallel to the $y$ axis. This implies that the disc rotates clockwise, and, as a consequence, the $z$-component of the disc's angular momentum and the disc's azimuthal velocity $v_{\Phi}$ are negative. Thus, negative $v_{\Phi}$ corresponds to prograde velocity rotation, and positive $v_{\Phi}$ to retrograde velocity rotation.

Finally, a note on the results that are presented in the following section of this work. As shown in Fig. 1, the sample studied in this paper is still relatively local, and most of it is restricted to distances of $2-3 \mathrm{kpc}$ from the Sun. It is thus natural to discuss how general the results are, and to what extent they could be generalised to other regions of the galactic disc. We started investigating this issue in the simulations presented 
by Jean-Baptiste et al. (2017, see Figs. 5 and 7, and Tables 3-5 in that paper). This work contains three simulations of a Milky Way-type galaxy accreting one or several satellites. We showed that, one Gyr after the accretion of a satellite, accreted stars are already very well-mixed, and their fraction, as well as their kinematic properties, do not significantly depend on the "solar volume" chosen, for solar volumes at the same Galactocentric distance. These simulations thus suggest that the results presented in this paper should be generalised to different regions of the galactic disc, at similar distances from the galactic centre. This similarity can be understood, because the dynamical times at the solar radius are relatively short: with a typical rotational period around the galactic centre of about $200 \mathrm{Myr}$, after one or few Gyrs, accreted stars can redistribute rather homogeneously in the disc. Therefore, for mergers which took place one Gyr ago or earlier, we expect that the mixing of accreted material at the solar radius is now complete.

\subsection{Kinematics versus abundances: mean relations}

Figure 2 (top-right panel and bottom panels) shows the mean azimuthal velocity, and the radial, vertical, and azimuthal velocity dispersions, respectively $v_{\Phi}, \sigma_{R}, \sigma_{Z}, \sigma_{\Phi}$, of stars in the sample in different loci in the $[\mathrm{Fe} / \mathrm{H}]-[\mathrm{Mg} / \mathrm{Fe}]$ plane. The $[\mathrm{Fe} / \mathrm{H}]$ and $[\mathrm{Mg} / \mathrm{Fe}]$ axes are divided into 25 bins. For each bin, the mean azimuthal velocity and velocity dispersion of stars in that bin were estimated, and only bins containing more than ten stars are shown (Fig. 2).

The result is clear that, in the $[\mathrm{Mg} / \mathrm{Fe}]-[\mathrm{Fe} / \mathrm{H}]$ plane, the low- $\alpha$ sequence of halo stars stands out as a distinct sequence in its kinematic properties with respect to both the high- $\alpha$ halo sequence and disc stars. As an example, part of this sequence shows a mean retrograde motion as high as $v_{\Phi}=50 \mathrm{~km} \mathrm{~s}^{-1}$. At $[\mathrm{Fe} / \mathrm{H}] \sim-1$, the vertical velocity dispersion, $\sigma_{Z}$, is about $50 \mathrm{~km} \mathrm{~s}^{-1}$ at the high- $\alpha$ end and increases to about $90 \mathrm{~km} \mathrm{~s}^{-1}$ at the low- $\alpha$ end. Figure 2 also shows that this oblique band of low $v_{\Phi}$ and high velocity dispersions in the $[\mathrm{Fe} / \mathrm{H}]-[\mathrm{Mg} / \mathrm{Fe}]$ plane, appears to extend also to metallicities $[\mathrm{Fe} / \mathrm{H}]>-1$. This implies that some accreted stars also have metallicities typical of disc stars. This is in agreement with the findings of Mackereth et al. (2019), who found that some high eccentricity, low- $\alpha$ stars also have $[\mathrm{Fe} / \mathrm{H}]>-1$ (see Fig. 1 in their paper). It is also in agreement with the studies of Nissen \& Schuster (2010), Hayes et al. (2018), who show that the chemical pattern of the low- $\alpha$ sequence extends up to at least $[\mathrm{Fe} / \mathrm{H}] \sim-0.8$. In Sect. 3.2, we will show that accreted stars within this same sequence can have metallicities as high as $[\mathrm{Fe} / \mathrm{H}] \sim-0.5$.

Figure 2 shows that at all $[\mathrm{Fe} / \mathrm{H}]$ where accreted, low- $\alpha$ stars are found, a rise in velocity dispersions is expected. This result naturally has a direct impact on galactic chemo-kinematic relations, as we show in Fig. 3, by slicing the $[\mathrm{Mg} / \mathrm{Fe}]-[\mathrm{Fe} / \mathrm{H}]$ in ten metallicity bins, ranging from $[\mathrm{Fe} / \mathrm{H}]=-2.1$ up to $[\mathrm{Fe} / \mathrm{H}]=$ 0.25 (see Appendix B for plots of the number of stars). For each bin, we considered all stars in the sample between $[\mathrm{Fe} / \mathrm{H}]_{\text {bin }} \pm$ $\Delta[\mathrm{Fe} / \mathrm{H}],[\mathrm{Fe} / \mathrm{H}]_{\text {bin }}$, with the central value of each metallicity bin given in the legend of Fig. 3 , and $\Delta[\mathrm{Fe} / \mathrm{H}]=0.125$ for all except the lowest metallicity bin, where $\Delta[\mathrm{Fe} / \mathrm{H}]=0.225$ to increase its significance. We only show bins that contain more than ten stars.

For the metallicity bins we found the following (Fig. 3): (1) for the high-metallicity bins, $[\mathrm{Fe} / \mathrm{H}]>-0.5$, there was an overall increase in all velocity dispersions with $[\mathrm{Mg} / \mathrm{Fe}]$, as is expected when moving from the cold kinematics of the thin disc, at low
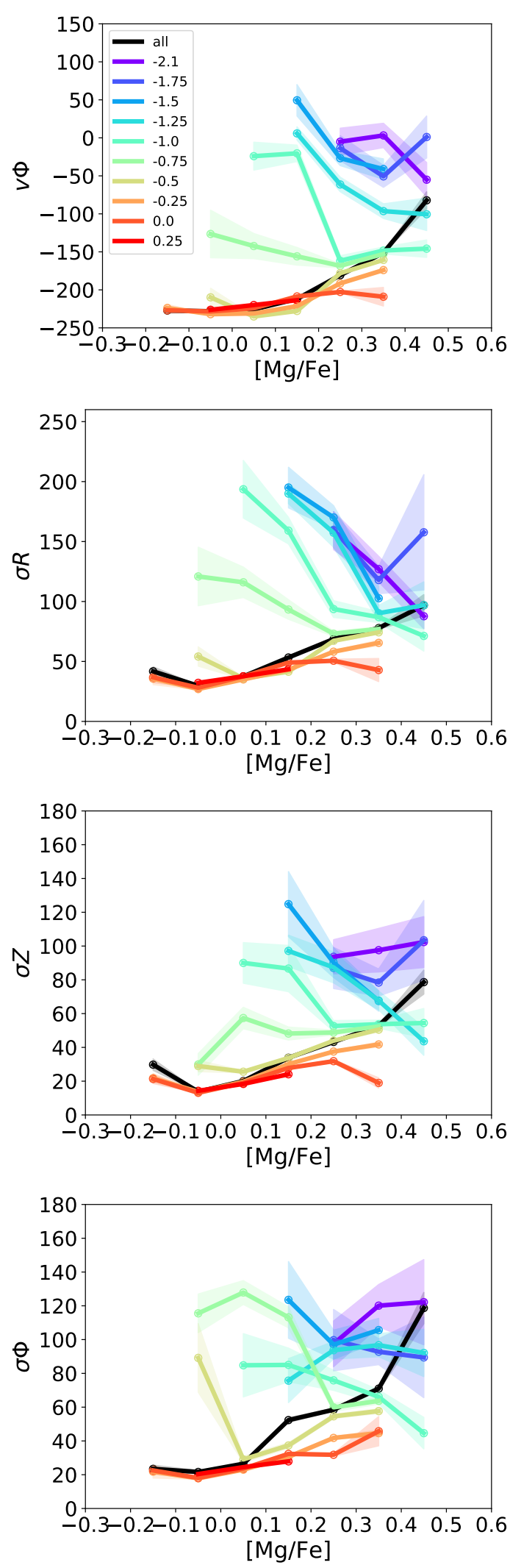

Fig. 3. From top to bottom: mean azimuthal velocity, radial, vertical and azimuthal velocity dispersion of stars, as a function of their $[\mathrm{Mg} / \mathrm{Fe}]$ ratio. In each panel, the relations are given for bins in $[\mathrm{Fe} / \mathrm{H}]$, as indicated in the legend in the top panel. The black curves show the corresponding relation, for the total sample not binned in $[\mathrm{Fe} / \mathrm{H}]$. The $1 \sigma$ uncertainty in each relation (coloured, shaded regions) is estimated through 1000 bootstrapped realisations. In all panels, only bins containing more than ten stars are shown. 
$[\mathrm{Mg} / \mathrm{Fe}]$, to the hot kinematics of the $\alpha$-enhanced thick $\operatorname{disc}^{3}$; (2) for $[\mathrm{Fe} / \mathrm{H}]=-0.5$ and -0.75 , the velocity dispersions no longer increased with $[\mathrm{Mg} / \mathrm{Fe}]$, but become rather constant with $[\mathrm{Mg} / \mathrm{Fe}] ;$ (3) at lower metallicities, the trend appears completely opposite to that observed at $[\mathrm{Fe} / \mathrm{H}]>-0.5$, and all components of the velocity dispersions decreased with $[\mathrm{Mg} / \mathrm{Fe}]$; (4) and finally, for $[\mathrm{Fe} / \mathrm{H}]<-1.75, \sigma_{Z}$ and $\sigma_{\Phi}$ did not show significant variations with $[\mathrm{Mg} / \mathrm{Fe}]$. It is also interesting that in the lowest metallicity bins, the maximum of the velocity dispersion was displaced to lower $[\mathrm{Mg} / \mathrm{Fe}]$ as $[\mathrm{Fe} / \mathrm{H}]$ increases. This behaviour was already noted by Minchev et al. (2014), and here we demonstrate that it is a natural signature of accreted stars whose abundances are characterised by an anti-correlation between $[\mathrm{Mg} / \mathrm{Fe}]$ and $[\mathrm{Fe} / \mathrm{H}]$.

Accreted stars also leave specific signatures in the $v_{\Phi^{-}}$ $[\mathrm{Mg} / \mathrm{Fe}]$ relations, for different $[\mathrm{Fe} / \mathrm{H}]$ (see Fig. 3). While the whole sample of stars shows a monotonic relation, with the mean rotation decreasing with $[\mathrm{Mg} / \mathrm{Fe}]$, different trends are found when stars are grouped in bins of $[\mathrm{Fe} / \mathrm{H}]$ : a monotonic relation of $v_{\Phi}$ with $[\mathrm{Mg} / \mathrm{Fe}]$ is found for $[\mathrm{Fe} / \mathrm{H}]>-0.5$; at $[\mathrm{Fe} / \mathrm{H}]=$ -0.5 , an upturn is observed at lowest $[\mathrm{Mg} / \mathrm{Fe}]$ end, with stars at $[\mathrm{Mg} / \mathrm{Fe}] \sim-0.05$ rotating as slow as stars at $[\mathrm{Mg} / \mathrm{Fe}] \sim 0.2$; the mean $v_{\Phi}$ appears nearly flat and independent of $[\mathrm{Mg} / \mathrm{Fe}]$ at $[\mathrm{Fe} / \mathrm{H}]=-0.75$, while the rotation increases with $[\mathrm{Mg} / \mathrm{Fe}]$, for $[\mathrm{Fe} / \mathrm{H}]<-0.75$. Particularly remarkable are the trends observed at $[\mathrm{Fe} / \mathrm{H}]=-1$. and $[\mathrm{Fe} / \mathrm{H}]=-1.25$ : here, the high $\alpha$-bins have a mean azimuthal velocity lagging behind that of the LSR by $100-150 \mathrm{~km} \mathrm{~s}^{-1}$, but have a mean prograde rotation, while the lowest $\alpha$-bins have a mean null or positive $v_{\Phi}$, indicative of a null or retrograde rotation. As for the velocity dispersion relations, we interpret the upturn observed at $[\mathrm{Fe} / \mathrm{H}]=-0.5$, and the features found at lower metallicities and low $[\mathrm{Mg} / \mathrm{Fe}]$, as the consequence of the presence of the accreted population, which is characterised by a mean null or slightly retrograde rotation (see Nissen \& Schuster 2010; Koppelman et al. 2018; Haywood et al. 2018; Helmi et al. 2018, and Sects. 3.2 and 3.3).

We conclude this section by presenting a similar analysis to that of Fig. 3, but this time slicing the $[\mathrm{Fe} / \mathrm{H}]-[\mathrm{Mg} / \mathrm{Fe}]$ plane in bins of $[\mathrm{Mg} / \mathrm{Fe}]$ (Fig. 4). Again, we investigate $v_{\Phi}, \sigma_{R}, \sigma_{Z}$ and $\sigma_{\Phi}$ relations as a function of $[\mathrm{Fe} / \mathrm{H}]$. For this, we sliced the $[\mathrm{Mg} / \mathrm{Fe}]-[\mathrm{Fe} / \mathrm{H}]$ into six bins of $[\mathrm{Mg} / \mathrm{Fe}]$, ranging from $[\mathrm{Mg} / \mathrm{Fe}]=-0.1$ up to $[\mathrm{Mg} / \mathrm{Fe}]=0.4$. For each bin, we consider all stars in the sample between $[\mathrm{Mg} / \mathrm{Fe}]_{\text {bin }} \pm \Delta[\mathrm{Mg} / \mathrm{Fe}]$, $[\mathrm{Mg} / \mathrm{Fe}]_{\text {bin }}$ to be the central value of the bin (see legend of Fig. 4). $\Delta[\mathrm{Mg} / \mathrm{Fe}]$ is 0.05 for all bins. The trends of $v_{\Phi}, \sigma_{R}$, $\sigma_{Z}$, and $\sigma_{\Phi}$ as a function of $[\mathrm{Fe} / \mathrm{H}]$ for the whole sample are as we expected - increasing rotation and decreasing velocity dispersions with increasing $[\mathrm{Fe} / \mathrm{H}]$. However, for different bins of $[\mathrm{Mg} / \mathrm{Fe}]$, these relations show specific characteristics. (1) For $[\mathrm{Fe} / \mathrm{H}]>-0.5$ and $[\mathrm{Mg} / \mathrm{Fe}]<0.1$, the rotation decreases as $[\mathrm{Fe} / \mathrm{H}]$ increases. This trend, also visible in the $v_{\Phi}$ relation (Fig. 2), has already been found in a number of studies, (Haywood 2008; Lee et al. 2011) and we confirm the existence of a positive gradient in the $v_{\Phi}-[\mathrm{Fe} / \mathrm{H}]$ relation for thindisc stars with $[\mathrm{Fe} / \mathrm{H}]>-0.5$. (2) For metallicities $[\mathrm{Fe} / \mathrm{H}] \leq$ -0.5 , there is a sharp increase in all the velocity dispersionsmetallicity relations, and also in the $v_{\Phi}-[\mathrm{Fe} / \mathrm{H}]$ relation, for all $[\mathrm{Mg} / \mathrm{Fe}]$ bins. The steepness of the relations at $[\mathrm{Fe} / \mathrm{H}] \leq$ -0.5 reaches a maximum for $[\mathrm{Mg} / \mathrm{Fe}]=0.0$, and decreases

\footnotetext{
3 The only exception to this trend is observed in the $\sigma_{z}$ versus $[\mathrm{Mg} / \mathrm{Fe}]$ relation, for the bin centred at $[\mathrm{Fe} / \mathrm{H}]=0$ and $[\mathrm{Mg} / \mathrm{Fe}]=0.35$, where one observes a drop in the value of $\sigma_{z}$ comparable to those found for $\alpha$-poor bins
}
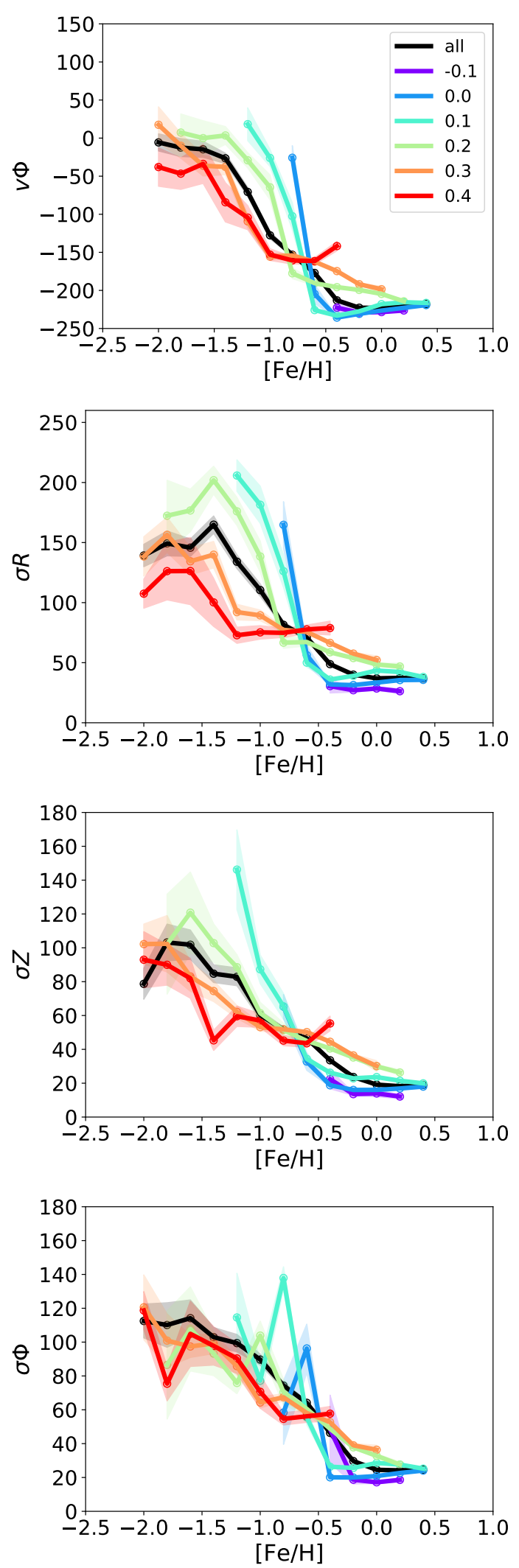

Fig. 4. Panels from top to bottom: mean azimuthal velocity, radial, vertical and azimuthal velocity dispersion of stars, as a function of their $[\mathrm{Fe} / \mathrm{H}]$ abundance. In each panel, the relations are for various bins in $[\mathrm{Mg} / \mathrm{Fe}]$, as indicated in the legend in the top panel. The black curves in each panel show the relation for the total sample. The $1 \sigma$ uncertainty in each relation (coloured region around each relation) is estimated using 1000 bootstrapped realizations. In all panels, only bins containing more than 10 stars are shown. 
with increasing $[\mathrm{Mg} / \mathrm{Fe}]$, and the metallicity where the increase becomes the most evident shifts towards lower metallicities as $[\mathrm{Mg} / \mathrm{Fe}]$ increases. In other words, for any given $[\mathrm{Fe} / \mathrm{H}]$ below -0.5 , the stellar kinematics become increasingly hotter as the relative $\alpha$-abundance ratio decrases. In particular, at $[\mathrm{Fe} / \mathrm{H}] \leq$ -1.5 , on one hand, high $[\mathrm{Mg} / \mathrm{Fe}]$ populations $([\mathrm{Mg} / \mathrm{Fe}]=0.4)$ have a mean prograde motion, while on the other hand, stars with $[\mathrm{Mg} / \mathrm{Fe}]=0.2$ have a mean retrograde motion. This is a clear indication that at these metallicities, at least two different populations, with different mean kinematic properties, co-exist.

Finally, we analyse the fraction of stars counter-rotating with respect to the galactic disc in the $[\mathrm{Fe} / \mathrm{H}]-[\mathrm{Mg} / \mathrm{Fe}]$ plane, as well as their fraction as a function of $[\mathrm{Mg} / \mathrm{Fe}]$ and $[\mathrm{Fe} / \mathrm{H}]$ (Fig. 5). Again, we sliced each of the two abundance planes in the same way we did previously (Figs. 3 and 4). The low- $[\mathrm{Mg} / \mathrm{Fe}]$ sequence also stands out as a distinct sequence for its high fraction of retrograde stars, significantly higher than that of the disc and halo at similar $[\mathrm{Fe} / \mathrm{H}]$, but higher $[\mathrm{Mg} / \mathrm{Fe}]$. While the fraction of counter-rotating $(\mathrm{CR})$ stars is typically less than $1 \%$ for disc stars with $[\mathrm{Fe} / \mathrm{H}]>-0.5$, we see that by $[\mathrm{Fe} / \mathrm{H}]=-0.5$ and $[\mathrm{Mg} / \mathrm{Fe}] \sim 0$, this fraction of CR-stars rises to more than $10 \%$, which is indicative of a significant population of accreted stars in this abundance range. As the metallicity decreases, the fraction of CR stars increases, especially for stars with low $[\mathrm{Mg} / \mathrm{Fe}]$ ratios. For example, at $[\mathrm{Fe} / \mathrm{H}]=-1$, the percentage of $\mathrm{CR}$ stars is low for the bin centred at $[\mathrm{Mg} / \mathrm{Fe}]=0.4$, but this fraction rises to more than $50 \%$ for $[\mathrm{Mg} / \mathrm{Fe}] \leq 0.2$. It is only for stars with $[\mathrm{Fe} / \mathrm{H}]<-1$ that the fraction of CR stars rises for the highest $[\mathrm{Mg} / \mathrm{Fe}]$ bin. This is obviously a sign that, below this metallicity, the accreted population significantly contaminates the high- $\alpha$ sequence of in-situ stars.

In all the analysis presented in this section, the statistical uncertainties in the relations have been estimated through a bootstrapping technique, with over 1000 realisations. We refer the reader to Appendix A for a comparison of these uncertainties, with those obtained by propagating the individual uncertainties on the observables (parallaxes, proper motions, and radial velocities).

\subsection{The fraction of in-situ and accreted stars across the $[\mathrm{Fe} / \mathrm{H}]-[\mathrm{Mg} / \mathrm{Fe}]$ plane}

In the previous section, we presented the chemo-kinematics relations of stars in the Gaia DR2-APOGEE sample, addressing the role accreted stars play in shaping these relations. We have already seen that at the extreme of the metal-poor thin-disc sequence, $[\mathrm{Fe} / \mathrm{H}]=-0.5$ and $[\mathrm{Mg} / \mathrm{Fe}]<0.1$, the presence of accreted stars can be demonstrated thanks to the signature they leave on the stellar kinematics. The question we want to address in this section concerns the relative fraction of accreted and in-situ stars for $[\mathrm{Fe} / \mathrm{H}] \leq-0.5$, and in particular, whether an in-situ population can also be identified at $[\mathrm{Fe} / \mathrm{H}] \leq-1.5$, in a region where the low and high- $\alpha$ sequences merge, and where no differentiation between accreted and in-situ populations seems possible only on the basis of elemental abundances.

To take a step further in our understanding of accreted and in-situ populations, we now present the velocity distributions of stars in different regions of the $[\mathrm{Fe} / \mathrm{H}]-[\mathrm{Mg} / \mathrm{Fe}]$ plane. Our aim is to use these distributions and the way they change across the abundance plane to weigh the fraction of accreted and in-situ material for different metallicity and $[\mathrm{Mg} / \mathrm{Fe}]$ bins. In doing so, we will also be naturally lead to discuss the origin of the in-situ population found across the $[\mathrm{Fe} / \mathrm{H}]-[\mathrm{Mg} / \mathrm{Fe}]$ plane. For this analysis, we have defined eight regions in the $[\mathrm{Fe} / \mathrm{H}]-[\mathrm{Mg} / \mathrm{Fe}]$ at the
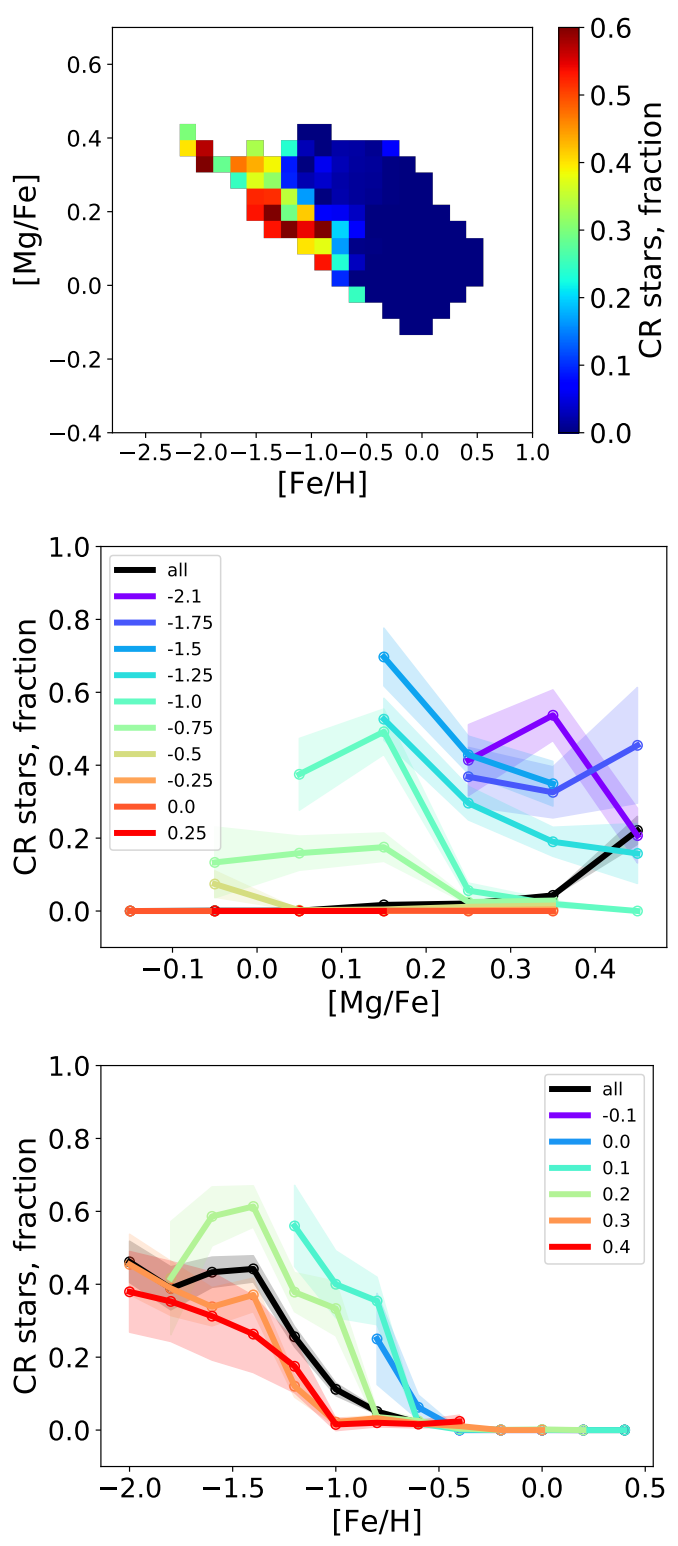

Fig. 5. Top panel: fraction of counter-rotating stars in $[\mathrm{Fe} / \mathrm{H}]-[\mathrm{Mg} / \mathrm{Fe}]$ plane. The pixel size is $0.152 \times 0.044$, and only pixels containing more than ten stars are shown. Middle panel: fraction of counter-rotating stars as a function of their $[\mathrm{Mg} / \mathrm{Fe}]$ ratio. In each panel, coloured curves indicate stars in specific bins of $[\mathrm{Fe} / \mathrm{H}]$, as indicated in the legend. The black curves show the corresponding relation for the total sample. Bottom panel: fraction of counter-rotating stars as a function of their $[\mathrm{Fe} / \mathrm{H}]$ ratio. In each panel, coloured curves indicate stars binned by their $[\mathrm{Mg} / \mathrm{Fe}]$ values (see legend in upper left-hand corner of the panel). The uncertainties were estimated using a bootstrapping procedure as previously described. Middle and bottom panels: only bins containing more than ten stars are shown.

crossroads between the thick disc, the metal-poor thin disc, and the low and high- $\alpha$ halo sequences as follows (see also, Fig. 6).

Three regions are chosen along the high- $[\mathrm{Mg} / \mathrm{Fe}]$ sequence: Region 1 contains stars in the $[\mathrm{Fe} / \mathrm{H}]-[\mathrm{Mg} / \mathrm{Fe}]=[[-2$, $-1.5],[0.25,0.35]]$ interval, Region 2 contains stars in the $[\mathrm{Fe} / \mathrm{H}]-[\mathrm{Mg} / \mathrm{Fe}]=[[-1.5,-1],[0.25,0.35]]$ interval, and Region 3 contains stars in the $[\mathrm{Fe} / \mathrm{H}]-[\mathrm{Mg} / \mathrm{Fe}]=[[-1 .,-0.5]$, $[0.25,0.35]]$ interval.

Three regions are chosen along the low- $[\mathrm{Mg} / \mathrm{Fe}]$ sequence of accreted stars: Region 5 contains stars in the $[\mathrm{Fe} / \mathrm{H}]-[\mathrm{Mg} / \mathrm{Fe}]=$ $[[-1.5,-1],[0.1,0.2]]$ interval, Region 6 contains stars in the 


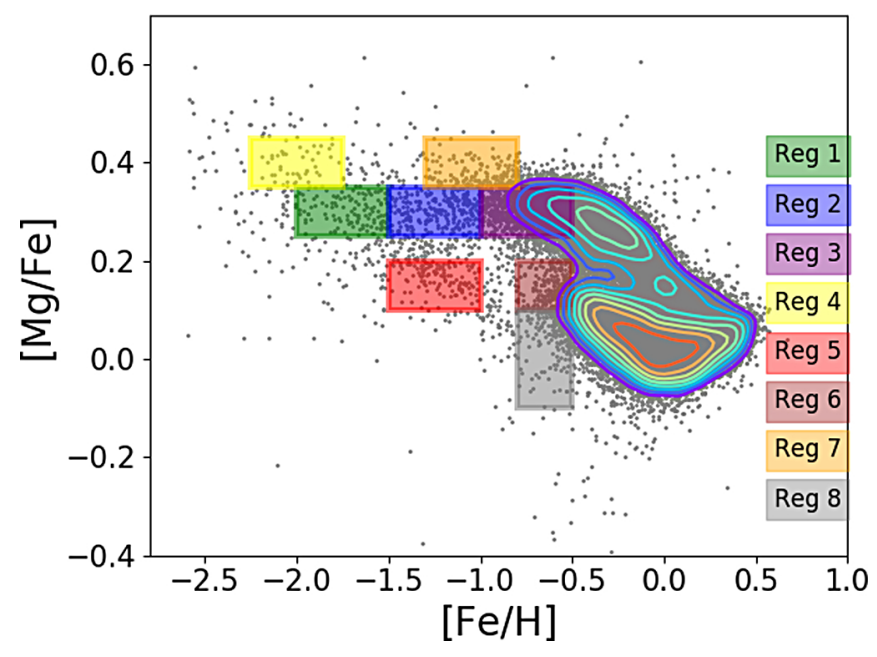

Fig. 6. Distribution of stars in $[\mathrm{Fe} / \mathrm{H}]-[\mathrm{Mg} / \mathrm{Fe}]$ plane with coloured lines indicating isodensity contours (see Fig. 2). We show eight regions, which are defined in Sect. 3.2, and are indicated with different colours, as shown on the left-hand axis of the panel.

$[\mathrm{Fe} / \mathrm{H}]-[\mathrm{Mg} / \mathrm{Fe}]=[[-0.8,-0.5],[0.1,0.2]]$ interval, thus at the upper edge of the metal-poor thin disc, and Region 8 contains stars with $[\mathrm{Fe} / \mathrm{H}]-[\mathrm{Mg} / \mathrm{Fe}]=[[-0.8,-0.5],[-0.1,0.1]]$, thus at the lower edge of the metal-poor thin disc and below it.

Finally, two regions were chosen at the upper edge of the high- $[\mathrm{Mg} / \mathrm{Fe}]$ sequence: Region 4 contains stars in the $[\mathrm{Fe} / \mathrm{H}]-[\mathrm{Mg} / \mathrm{Fe}]=[[-2.25,-1.75],[0.35,0.45]]$ interval, and Region 7 contains stars in the $[\mathrm{Fe} / \mathrm{H}]-[\mathrm{Mg} / \mathrm{Fe}]=$ $[[-1.3,-0.8],[0.35,0.45]]$ interval. Despite these two regions containing fewer stars than the previous ones, they were chosen to tentatively probe the in-situ/accreted content also at these levels of $[\mathrm{Mg} / \mathrm{Fe}]$-enhancement.

Two of the eight regions are particularly important for this study, as they serve as fiducial regions with which to compare and analyse all of the regions. One is Region 3, which is not contaminated by the accreted sequence of stars with low- $[\mathrm{Mg} / \mathrm{Fe}]$, and which we will use as the reference for in-situ stars belonging to the galactic thick disc. The other is Region 5, which lies on the elemental-abundance defined sequence for stars that were accreted. As we will see in the following section, stars in Region 5 have peculiar kinematics, clearly different from those of Region 3. This suggests that the properties of stars in Region 5 can be used as a reference for the accreted sequence. We caution that even if we refer to Region 3 as a reference for the thick disc, it also contains a tail in its distribution of properties, which includes stars that are rotating more slowly, and even counterrotating relative to disc stars. Despite not being contaminated by the accreted sequence of low- $[\mathrm{Mg} / \mathrm{Fe}]$ stars, the kinematics of stars in this region has indeed been significantly affected by this accretion (see Sect. 3.3).

In Fig. 7, we show the distributions of the azimuthal velocities, $v_{\Phi}$, for stars in each of these regions. For each region, the medians of the distributions are also shown. Uncertainties in this and in the following distributions (see Figs. 8 and 9) were calculated by bootstrapping the sample, and for each bootstrap realisation, we have also taken into account the individual uncertainties in the velocities using a Monte-Carlo sampling. We explicitly considered the individual uncertainties to make sure that the distributions are also robust for stars with halo kinematics, whose individual uncertainties, on average, are higher than those of disc stars (see Fig. A.2).
The "pure" accreted region (Region 5) is characterised by an average weak retrograde distribution peaking $v_{\Phi}=2.5 \mathrm{~km} \mathrm{~s}^{-1}$ (median), while the "pure" thick-disc region (Region 3 ) is characterised by an average prograde rotation peaking at $v_{\Phi}=$ $-168.1 \mathrm{~km} \mathrm{~s}^{-1}$ (median). Moving from Region 3 to Region 2, and then to Region 1, and thus moving to lower metallicities, at constant $[\mathrm{Mg} / \mathrm{Fe}]$ ratio, the $v_{\Phi}$ distribution can be qualitatively described as the weighted sum of the distributions of Regions 3 and 5. Therefore, it can be stated that the combination of thick-disc and accreted stars and the stellar population rotate more slowly, on average. The medians of the distribution indeed increase to $v_{\Phi}=-109.4 \mathrm{~km} \mathrm{~s}^{-1}$ in Region 2 , and to $v_{\Phi}=$ $-23.8 \mathrm{~km} \mathrm{~s}^{-1}$ (median) in Region 1. However, the comparison of the distributions in these three regions shows that the rotation decreases not because of a decrease in the rotation of the thick disc at lower metallicities, but, in fact, the purple line, which indicates the median of the distribution in Region 3, always coincides with the fast-rotating peak of the distributions of Region 2 and Region 1 . The decrease in the median rotation with decreasing $[\mathrm{Fe} / \mathrm{H}]$, is most likely due to an increase in the fraction of stars with null or retrograde motion. The evidence for this is the increasing fraction of stars in Region 2 and Region 1 with $v_{\Phi}$ equal to or higher than the median $v_{\Phi}$ value of Region 5. As we demonstrate in the following section, the distribution of $v_{\Phi}$ in Regions 1 and 2 can be understood as a weighted sum of the $v_{\Phi}$ distributions of Regions 3 and 5, with the relative fraction of the accreted stars increasing when moving from Region 2 to 1. It is natural to make use of this modulation of $v_{\Phi}$ distributions to try to derive the fraction of accreted and in-situ stars, on the high- $[\mathrm{Mg} / \mathrm{Fe}]$ sequence, as a function of $[\mathrm{Fe} / \mathrm{H}]$. We caution the reader that the approach used here is simple and should only be thought of as a first attempt to estimate the relative fractions of these two populations in different regions of the abundance plane.

In Fig. 8, we show the histograms of the azimuthal velocities $v_{\Phi}$ for Regions 1 and 2, this time overlaying the $v_{\Phi}$ distributions of Region 3 on each of them, which we consider as consisting purely of thick-disc stars, and Region 5, which we consider as consisting purely of accreted stars. To compare the distributions of Regions 3 and 5 to those of Regions 1 and 2, we applied a $\chi^{2}$-minimisation to find the normalisation constants for the distributions of Regions 3 and 5, which minimise the quadratic difference between the normalised sum of Regions 3 and 5, and the distribution of Regions 1 (and 2).

This way, we can estimate the fractional contribution of Regions 3 and 5, to Regions 1 and 2, respectively, leading to the following results. For Region 2, which includes stars with $[\mathrm{Fe} / \mathrm{H}]-[\mathrm{Mg} / \mathrm{Fe}]=[[-1.5,-1],[0.25,0.35]]$, we estimate the fraction of accreted stars to be about $25 \%$ of the total number of stars in these abundance intervals. This fraction increases to about $70 \%$ in Region 1, which includes all stars in the sample with $[\mathrm{Fe} / \mathrm{H}]-[\mathrm{Mg} / \mathrm{Fe}]=[[-2 .,-1.5],[0.25,0.35]]$. To further test the null hypothesis that the distributions in Regions 1 and 2 can be described as the sum of the distributions of stars in the reference thick-disc region, and of stars in the accreted region, we ran a Kolmogorov-Smirnov test, finding KS-statistics and a $p$-value equal to 0.08 and 0.95 for Region 1 , and 0.12 and 0.91 for Region 2 . These values show that the hypothesis that Regions 1 and 2 are made simply of stars of the thick disc and of accreted material cannot confidently be rejected.

Before discussing the $v_{\Phi}$ distributions in other regions of the $[\mathrm{Fe} / \mathrm{H}]-[\mathrm{Mg} / \mathrm{Fe}]$ plane, we would like to comment on the excess of stars at $v_{\Phi} \sim-100 \mathrm{~km} \mathrm{~s}^{-1}$ that appears when comparing the distribution of stars in Region 2 with the normalised 

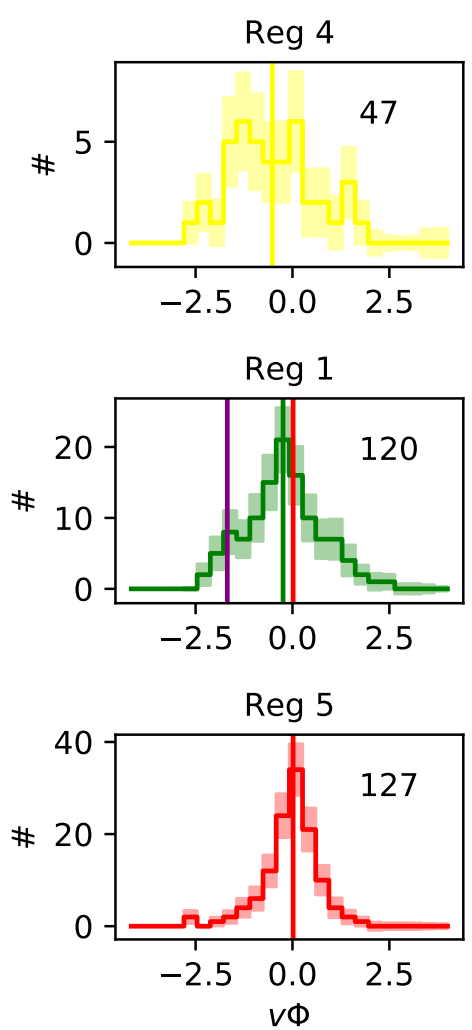

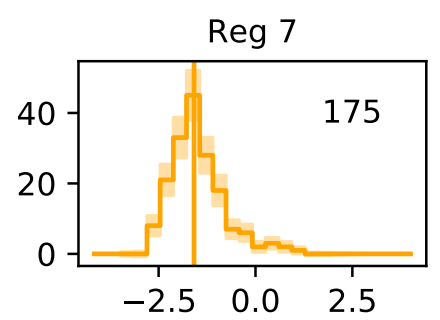

Reg 2
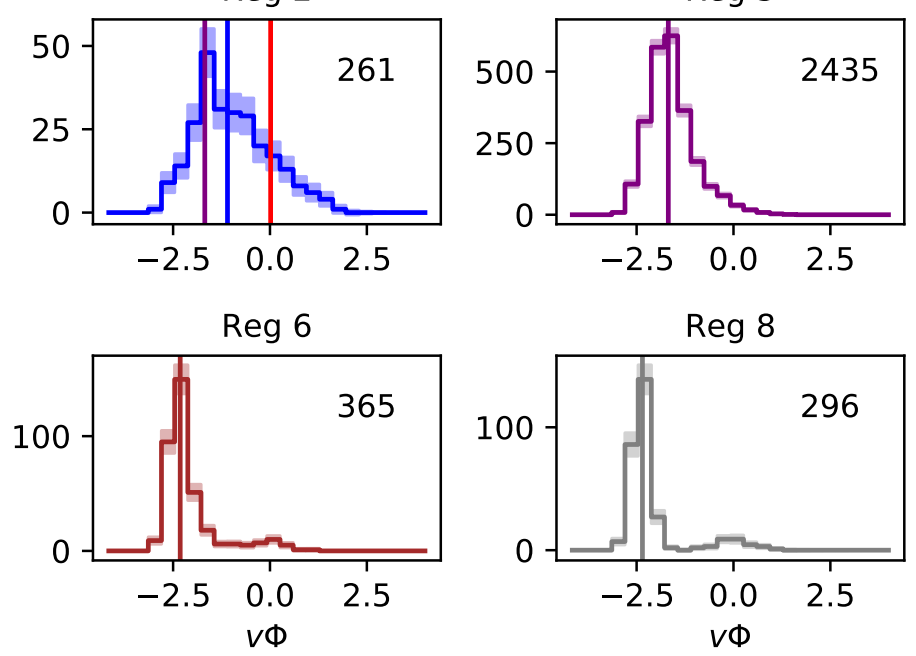

Fig. 7. Absolute distribution of azimuthal velocities, $v_{\Phi}$, for stars in eight regions shown in Fig. 6 (see Sect. 3.2). In all panels: velocities are in units of $100 \mathrm{~km} \mathrm{~s}^{-1}$, and the solid coloured lines correspond to the medians of the distributions. In Regions 1 and 2, the solid purple and red lines correspond to the medians of the $v_{\Phi}$ distributions in Regions 3 and 5, respectively. The number of stars in each region is provided in the upper-left corner of each panel. The $1 \sigma$ uncertainties (coloured, shaded regions) have been estimated by taking into account both the statistical uncertainty, through 1000 bootstrapped realisations, and the individual uncertainties propagated from the observables.

sum of Regions 3 and 5 (see Fig. 8, bottom panel). The current uncertainties make this excess of stars not statistically significant (at these values of $v_{\Phi}$, the distributions are compatible within $2 \sigma$.) However, if this excess is confirmed by further studies, some hypotheses about its origin can be proposed: (1) it could be mainly made of accreted stars belonging to the same major accretion event that makes the majority of stars found in Region 5 (in Jean-Baptiste et al. (2017) we showed that accreted stars that come from the same parent satellite do not necessarily all have the same kinematic properties, the latter depending on the time they were stripped from the parent satellite, whether at the first passage or later); (2) it could still be part of the thick disc, meaning that this excess could still have, for the majority, an in-situ origin, and this would imply that the rotation of the thick disc, at these metallicities, would be slightly lower than that of thick disc stars at higher $[\mathrm{Fe} / \mathrm{H}]$. In all cases, we emphasise that this excess of stars - if real-only represents about $10 \%$ of stars in Region 2 , that is less than $5 \%$ of all stars with $[\mathrm{Fe} / \mathrm{H}] \leq-1$ in our sample, and thus does not impact our conclusions.

Because of the similarity of its $v_{\Phi}$ distribution with that of Region 3, and because of its location in the $[\mathrm{Fe} / \mathrm{H}]-[\mathrm{Mg} / \mathrm{Fe}]$ plane, we conclude that Region 7 is not contaminated by accreted stars, while some accreted stars are found in Regions 6 and Region 8. While the vast majority of stars in these regions have prograde motions, a secondary, weak peak at positive $v_{\Phi}$ is visible in both distributions. This is further evidence, together with the analysis presented in Sect. 3.1, that accreted stars are present up to the edge of the metal-poor thin $\operatorname{disc},[\mathrm{Fe} / \mathrm{H}] \sim$ -0.5 , at the level of few percent. We do not draw any conclusions about Region 4 because of very poor statistics.
By summing the contribution of in-situ stars in the Regions 1, 2 and 5, and by comparing it to the total number of stars in those regions, we can derive a rough estimate of their fraction in our sample for $[\mathrm{Fe} / \mathrm{H}] \leq-1$. We estimate that the contribution of in-situ stars is $\sim 40-45 \%$. While we must be cautious about the actual values, our estimate indicates that thickdisc stars should constitute a non-negligible fraction of the stars found at metallicities $-2 \leq[\mathrm{Fe} / \mathrm{H}] \leq-1$. These stars constitute the metal-poor tail of the thick disc, the so-called metal-weak thick disc (see Sect. 4).

Finally, the distributions of radial velocities, $v_{R}$, shown in Fig. 9 reinforce our previous conclusions. Region 5, which we take as the reference for a region dominated by accreted stars, has most of its stars in two high-velocity peaks $\left(\left|v_{R}\right|>\right.$ $200 \mathrm{~km} \mathrm{~s}^{-1}$ ). The regions that, according to our analysis, are contaminated or dominated by accreted stars, Regions 1, 2, 6, and 8 , all show a tail in their distribution of stars with high radial velocities, $\left|v_{R}\right|>200 \mathrm{~km} \mathrm{~s}^{-1}$. These high radial-velocity stars are clearly visible in Regions 1 and 2, and in less significant numbers in Regions 6 and 8.

In the $v_{R}-v_{\Phi}$ plane (Fig. 10, top panel), the different kinematics of accreted and in-situ populations are even more evident. While in Region 5, stars are distributed along a horizontal line of $v_{\Phi} \approx 0 \mathrm{~km} \mathrm{~s}^{-1}$, with absolute values of $v_{R}$ as high as $400 \mathrm{~km} \mathrm{~s}^{-1}$, in Regions 6 and 8, most of the stars are grouped in a clump of fast prograde rotating stars, with $v_{\Phi} \leq-200 \mathrm{~km} \mathrm{~s}^{-1}$. Only few tens of stars are found in the region of accreted stars (i.e. having kinematics like stars in Region 5). In Regions 1 and 2, both populations co-exist, while the distribution of stars of Region 3 in the $v_{R}-v_{\Phi}$ does not show any clear contamination from accreted 

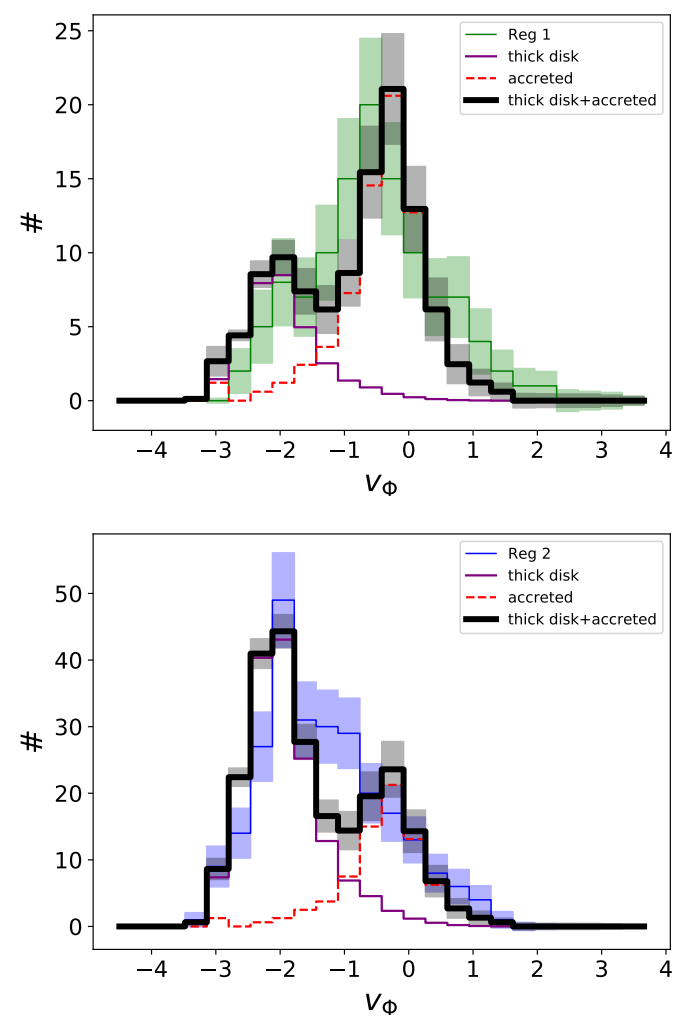

Fig. 8. Absolute distribution of the azimuthal velocities, $v_{\Phi}$, for stars in Regions 1 (top panel) and 2 (bottom panel). Also shown are histograms of distributions of Regions 3 and 5 (solid purple and dashed red lines, respectively), normalised as described in the text, and their sum (thick black lines). The $1 \sigma$ uncertainty in the distribution of stars in Regions 1 and 2 are shown, respectively, by green and blue shaded regions (top and bottom panels), and the uncertainty in the normalised sum of Regions 3 and 5 is shown by a grey shaded area. All uncertainties have been estimated by taking into account both the statistical uncertainty, through 1000 bootstrapped realisations, and the individual uncertainties propagated from the observables. In all panels: velocities are in units of $100 \mathrm{~km} \mathrm{~s}^{-1}$.

stars. Also, in the $v_{\Phi}-v_{Z}$ plane (see Fig. 10, bottom panel), the main kinematic populations can be easily distinguished (e.g. Regions 6,8).

\subsection{Age-dating the accretion event}

When did this accretion event occur? If the fusion of the Milky Way with another galaxy happened, and some of the stars that were heated belong to already formed components of the Milky Way, then it should be possible to estimate when the merger occurred. The accretion of satellites onto Milky Way-type galaxies has been extensively studied through simulations over the last decades. Two main results of said simulations are interesting within this context: (1) the disc of the Milky Way must have heated during the accretion - that is its radial, vertical and azimuthal velocity dispersions must have increased by an amount that depends on the inclination of the satellites, its mass, and on the gas content of the main galaxy at the time of the accretion (Quinn et al. 1993; Walker et al. 1996; Velazquez \& White 1999; Font et al. 2001; Benson et al. 2004; Moster et al. 2010; Villalobos \& Helmi 2008; Kazantzidis et al. 2008; Villalobos \& Helmi 2009; Qu et al. 2010, 2011b,a; House et al. 2011) - and, importantly, the disc stars that were impacted by the merger should slow down (Qu et al. 2010); (2) there should be a significant overlap in the kinematics of the accreted stars and the in-situ stars present in the early Milky Way disc at the time of the merger. For example, a merger with a mass ratio of about 1:10, possibly similar to the mass ratio of the accreted satellite studied here, and which may define the nature of the low- $\alpha$ sequence at the time of its accretion (Haywood et al. 2018; Helmi et al. 2018), is expected to slow the rotation of some of this stars in the disc significantly (Jean-Baptiste et al. 2017).

In Figs. 11-13, we now make use of these arguments to look for signatures of the kinematic heating of the Milky Way disc that must have been generated at the time when stars were accreted onto the Galaxy. We start by showing the distribution in the $v_{\Phi}-[\mathrm{Fe} / \mathrm{H}]$ of all sample stars (Fig. 11). This is the same plane already shown in Fig. 4, but here we can see the gain in looking at the entire distribution of stars, instead of looking at the simple mean relations. Stars redistribute in this plane in a shoelike shape, with the tip of the shoe at high metallicities, $[\mathrm{Fe} / \mathrm{H}] \geq$ -0.2 , and the instep of the shoe at $[\mathrm{Fe} / \mathrm{H}] \leq-0.3$. Moving from the tip to the instep, $[\mathrm{Mg} / \mathrm{Fe}]$ increases, as expected for populations whose $[\mathrm{Fe} / \mathrm{H}]$ decreases. Also, the accreted sequence stands out clearly, as a sequence of $\left\langle v_{\Phi}\right\rangle \sim 0 \mathrm{~km} \mathrm{~s}^{-1}$, and lower $[\mathrm{Mg} / \mathrm{Fe}]$ values compared to the surrounding (red/brown in the plot) stars. The striking feature in this plot, however, is the rapid decline of the rotation (i.e. steep rise of $v_{\Phi}$ ) with decreasing $[\mathrm{Fe} / \mathrm{H}]$, for $[\mathrm{Fe} / \mathrm{H}] \leq-0.3$, something not clearly visible in the mean relation shown in Fig. 4, where the decline in the mean rotation starts at lower $[\mathrm{Fe} / \mathrm{H}]$. To better understand this trend, we show, in Fig. 12, the same $v_{\Phi}-[\mathrm{Fe} / \mathrm{H}]$ plane, but this time for stars binned by their $[\mathrm{Mg} / \mathrm{Fe}]$ ratios. The amplitude of the bins and their mean values are the same as in Fig. 4. For $[\mathrm{Mg} / \mathrm{Fe}]=-0.1,0$. and 0.1 , we can clearly distinguish two separated groups in their kinematics, one with $\left\langle v_{\Phi}\right\rangle \sim 0 \mathrm{~km} \mathrm{~s}^{-1}$, and the second one with prograde rotation (i.e. negative $v_{\Phi}$ ). At $[\mathrm{Mg} / \mathrm{Fe}]=0.2$, however, the separation between the two groups is no longer evident, and stars of the in-situ sequence with null or retrograde rotation appear at $[\mathrm{Fe} / \mathrm{H}] \sim-0.3$. The same thing is evident at $[\mathrm{Mg} / \mathrm{Fe}]=0.3$, where, again, one sees clearly that the in-situ population extends up to positive $v_{\Phi}$ (retrograde motions). At $[\mathrm{Mg} / \mathrm{Fe}]=0.4$, the distinction between the two groups is no longer evident in this plane but the limited number of stars in this bin does not allow us to draw any robust conclusions. However, the remarkable result we glean from this figure is that, while at $[\mathrm{Mg} / \mathrm{Fe}]<0.2$, the group with prograde rotation has distinct $v_{\Phi}$ from that of accreted stars, at $[\mathrm{Mg} / \mathrm{Fe}] \gtrsim 0.2$, a tail of zero or positive $v_{\Phi}-$ null or retrograde motions - appears among the metal-rich stars, $[\mathrm{Fe} / \mathrm{H}]>-1$.

To investigate this change further in the $v_{\Phi}$ properties of stars with $[\mathrm{Mg} / \mathrm{Fe}]$, in Fig. 13, we present the same analysis as in Fig. 12, but this time only for the $0.15 \leq[\mathrm{Mg} / \mathrm{Fe}] \leq 0.35$ interval, which has been divided in four bins. We can clearly see a plume of stars extending from $v_{\Phi}$, which is typical of the thick disc, to zero and positive $v_{\Phi}$, reaching values of the azimuthal velocity typical of the accreted component. This plume is not evident at $[\mathrm{Mg} / \mathrm{Fe}]<0.2$, appears at $0.2 \leq[\mathrm{Mg} / \mathrm{Fe}] \leq 0.25$ and $[\mathrm{Fe} / \mathrm{H}] \sim-0.3$, and is still present at higher $[\mathrm{Mg} / \mathrm{Fe}]$ ratios and slightly lower metallicities. We interpret this excess of stars with thick-disc abundances but accreted-like $v_{\Phi}$ as the signature of the heating of the early Milky Way disc by the satellite. To show that this kinematic heating does not only affect the azimuthal velocities, but also the radial and vertical motions, we show in Fig. 14 the distribution in the Toomre diagram - that is in the $v_{\Phi}-\sqrt{v_{R}^{2}+v_{Z}^{2}}-$ of stars binned according to their $[\mathrm{Mg} / \mathrm{Fe}]$ abundance, as in Fig. 12. In each panel of the plot, the dashed curve represents the locus of stars with 

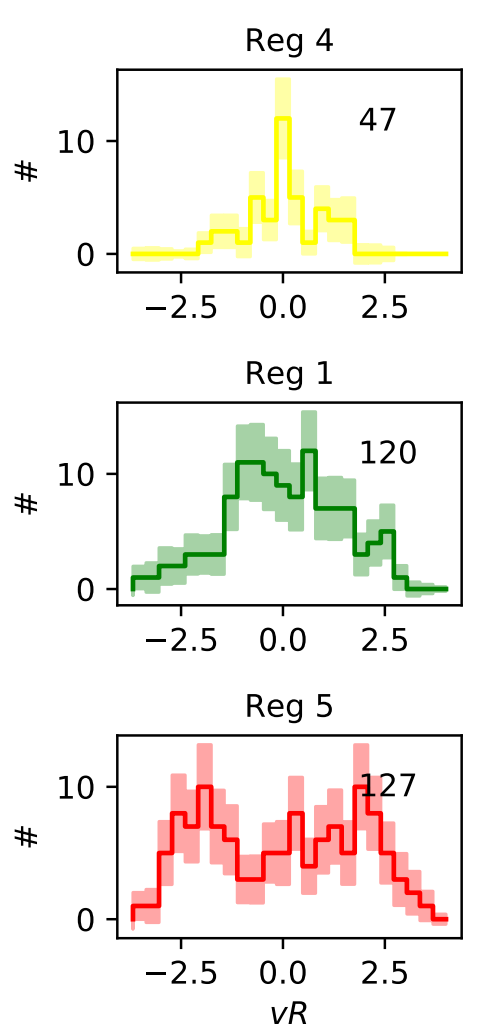
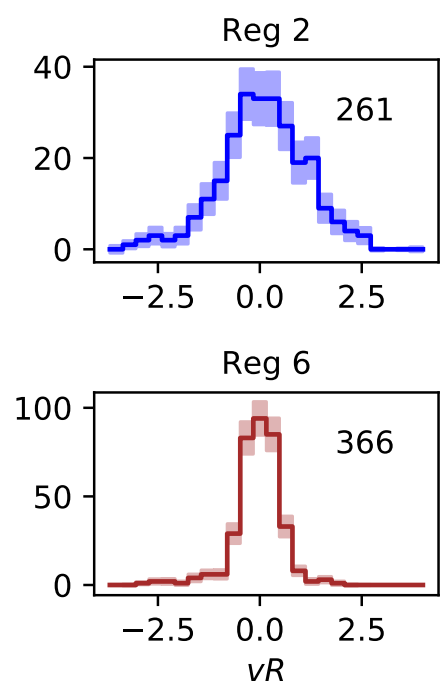
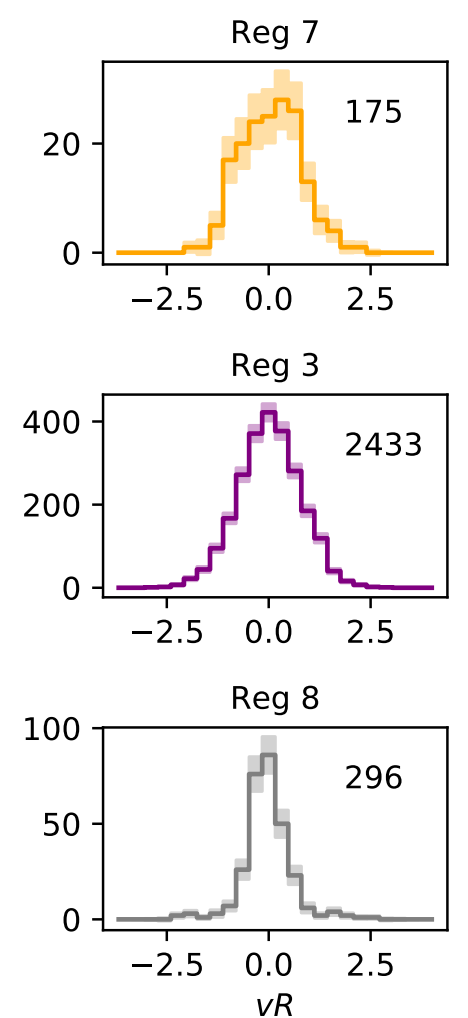

Fig. 9. Absolute distribution of the radial velocities, $v_{R}$, for stars in eight regions defined in Fig. 6 (see Sect. 3.2). In all panels, velocities are in units of $100 \mathrm{~km} \mathrm{~s}^{-1}$. The number of stars in each region is given at the upper-left of each panel. The $1 \sigma$ uncertainties (coloured, shaded regions) were estimated by taking into account both the statistical uncertainty, through 1000 bootstrapped realisations, and the individual uncertainties propagated from the observables.

$\sqrt{\left(v_{\Phi}-v_{\mathrm{LSR}}\right)^{2}+v_{R}^{2}+v_{Z}^{2}}=180 \mathrm{~km} \mathrm{~s}^{-1}$, commonly used as a separator between thick-disc and halo kinematics (see, for example Nissen \& Schuster 2010), and the colour code for the metallicity, $[\mathrm{Fe} / \mathrm{H}]$. For $[\mathrm{Mg} / \mathrm{Fe}] \leq 0.1$, two distinct groups appear: a sequence of metal-poor stars (with typical $[\mathrm{Fe} / \mathrm{H}] \leq-0.5$ ), which redistributes vertically in this plane, along the null $v_{\Phi}$ line, and which is thus characterised by halo-like kinematics; a sequence (with $[\mathrm{Fe} / \mathrm{H}] \geq-1$ ) - the vast majority of stars with $\sqrt{\left(v_{\Phi}-v_{\mathrm{LSR}}\right)^{2}+v_{R}^{2}+v_{Z}^{2}}<180 \mathrm{~km} \mathrm{~s}^{-1}$, and thus with thin and thick disc-like kinematics. The first of the two sequences is made of accreted stars, as discussed in the previous sections, and also shown by Haywood et al. (2018) and Helmi et al. (2018). At $[\mathrm{Mg} / \mathrm{Fe}]=0.2$, however, the distinction between the two sequences becomes less evident, and at $[\mathrm{Mg} / \mathrm{Fe}]>0.2$, the kinematic borders of the two sequences can no longer be distinguished. In particular, clearly, a sequence of stars with thickdisc metallicities but halo kinematics is found at $[\mathrm{Mg} / \mathrm{Fe}]=0.2$ (see also Fig. 15). These stars do not only overlap with the accreted sequence in $v_{\Phi}$, as previously shown, but also in their coupled radial and vertical motions. This overlap of kinematic properties of accreted and in-situ stars, predicted by simulations (Jean-Baptiste et al. 2017), is thus clearly present at the high$[\mathrm{Mg} / \mathrm{Fe}]$ end of the sample, and constitutes the smoking gun of the accretion event experienced by the Galaxy in its early evolution, and of the resulting kinematic heating.

Having defined the lower $[\mathrm{Mg} / \mathrm{Fe}]$ limit of the heating event, we can now convert the abundance ratio into an age, in order to age-date the merger. For this, we need to quantify the evolution of the $[\mathrm{Mg} / \mathrm{Fe}]$ ratio with age. Because our cross-matched Gaia DR2-APOGEE sample is made of giants (we remind the reader that only stars with $1<\log (g)<3.5$ have been retained for this analysis), we cannot date these stars directly by isochrone fitting techniques, as done, for example, in Haywood et al. (2013). However, as done in Haywood et al. (2013), we derive ages for a subsample of 273 dwarf stars from the Adibekyan et al. (2012) sample, retaining only stars with $M v<4.5$, the only difference with the work of Haywood et al. (2013) being that here we make use of parallaxes from Gaia DR2. We refer the reader to Haywood et al. (2013) for all the details concerning the adopted methodology for the age determination (bayesian method, set of isochrones, estimates on the age errors), and to a more extensive work in preparation (Haywood et al, in prep.) for the analysis and discussion of ages with Gaia DR2. In Fig. 16, we report the derived age- $[\mathrm{Mg} / \mathrm{Fe}]$ relation for this subsample ${ }^{4}$. We emphasise that, even if the sample used to estimate the ages is local, meaning it is confined to stars at few hundred parsecs from the Sun, these stars come from different parts of the disc. We have indeed shown in previous works that the distribution of their pericentres (Haywood et al. 2015, Fig. 4) and apocentres (Snaith et al. 2015, Fig. 3) is very broad, in particular for stars at the $[\mathrm{Mg} / \mathrm{Fe}]$ levels relevant to the dating of the accretion. The large extent of their orbits implies that these stars (currently observed at the solar vicinity) are indeed representative of a large portion of the inner disc.

4 Note that in this plot, the error bars on the magnesium abundances are fixed to 0.03 dex for all stars. This error comes from the estimate given in Adibekyan et al. (2012) for solar type stars. Cooler and hotter stars have mean uncertainties on a magnesium abundance of 0.07 and 0.05 dex (see their table 3). Three stars (HD 124785, HD 141597 and CD -436810) are identified as "young", alpha-rich stars, having magnesium, silicon and titanium abundances higher than 0.15 dex and ages under eight Gyr. 

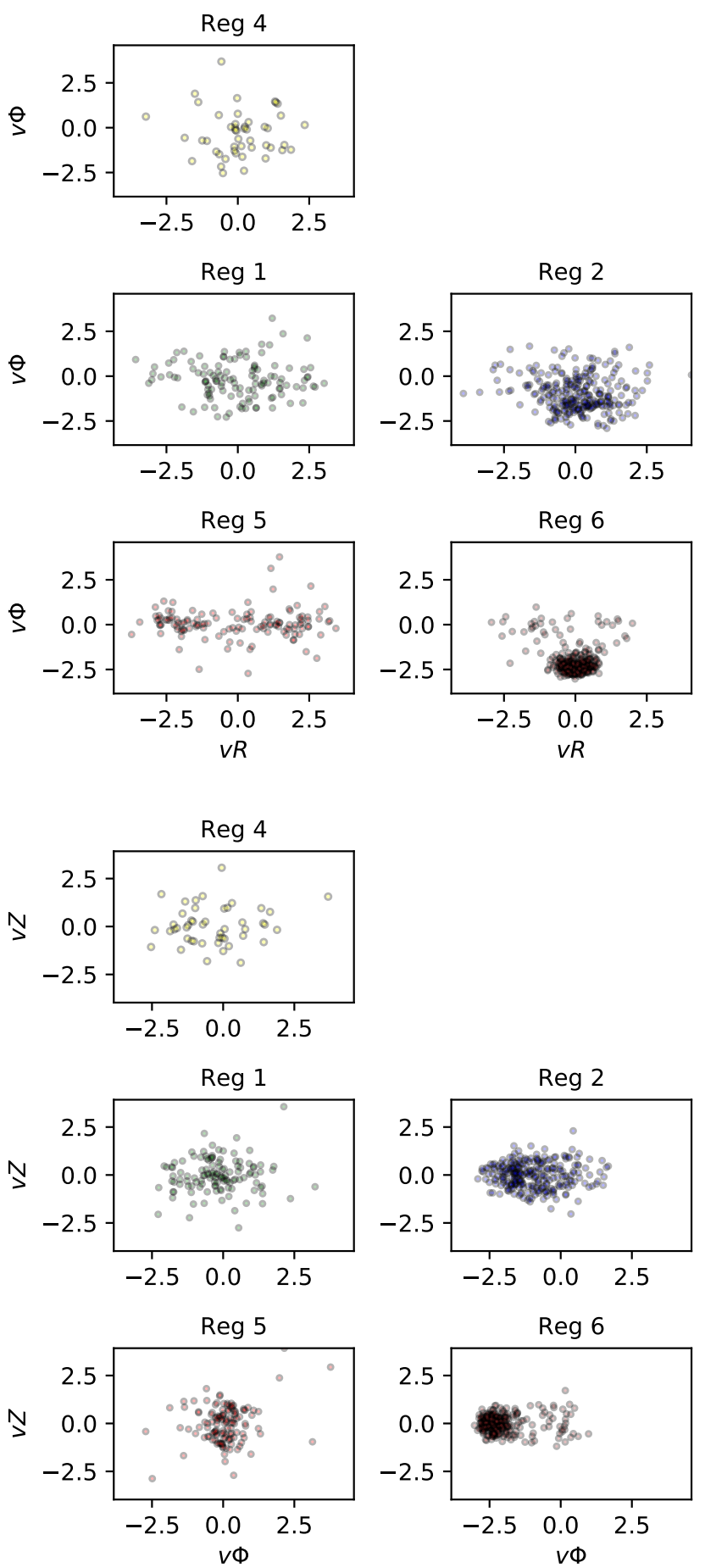

For a $[\mathrm{Mg} / \mathrm{Fe}]$ interval equal to $[0.2,0.25]$, which corresponds to the end of the disc heating phase, the corresponding age interval is nine $-11 \mathrm{Gyr}^{5}$. We emphasise that this estimate coincides with the end of the satellite merging process - or better, with the time when the kinematic heating caused by the

\footnotetext{
5 The biggest uncertainty on this estimate comes from possible systematics between the magnesium abundance scales of APOGEE and Adibekyan et al. (2012). There are eight stars in common between these two surveys, which have magnesium abundances between 0.13 and 0.36 . The mean of the differences between the $[\mathrm{Mg} / \mathrm{Fe}]$ abundances of the two samples for these eight stars is 0.002 dex (and a dispersion around this value of $0.059 \mathrm{dex}$ ), suggesting (although the strength of the evidence is limited because of the small number of stars) that there is no serious bias between the two abundance scales.
}
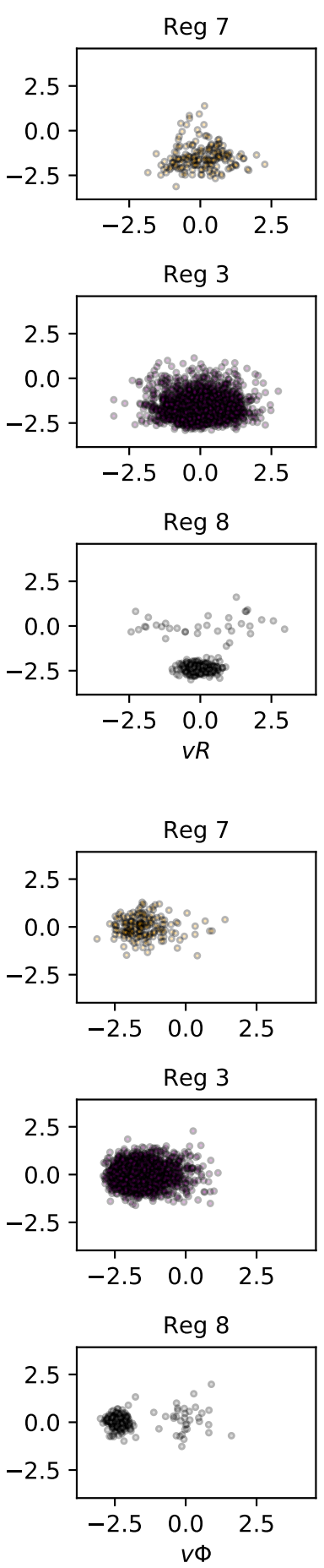

Fig. 10. Distribution of stars in $v_{R}-v_{\Phi}$ plane (top panels) and in $v_{\Phi}-v_{Z}$ plane (bottom panels) for eight regions defined in Fig. 6 (see Sect. 3.2). In all plots, velocities are in units of $100 \mathrm{~km} \mathrm{~s}^{-1}$.

decaying satellite to the early Milky Way disc became negligible. According to the N-body models by Qu et al. (2011a), indeed, the heating of the early galactic disc may have already started at the first close passage of the satellite to the Milky Way, and we can expect the heating to be significant in the first phases of the accretion (see Fig. 3 in their paper) and continued up to the final phases of the accretion.

\section{Discussion}

\subsection{The overlapping borders of galactic stellar populations: revising Minchev et al. (2014) results}

In the previous section, we saw that discs and halo populations can overlap both in chemical and kinematic spaces: 


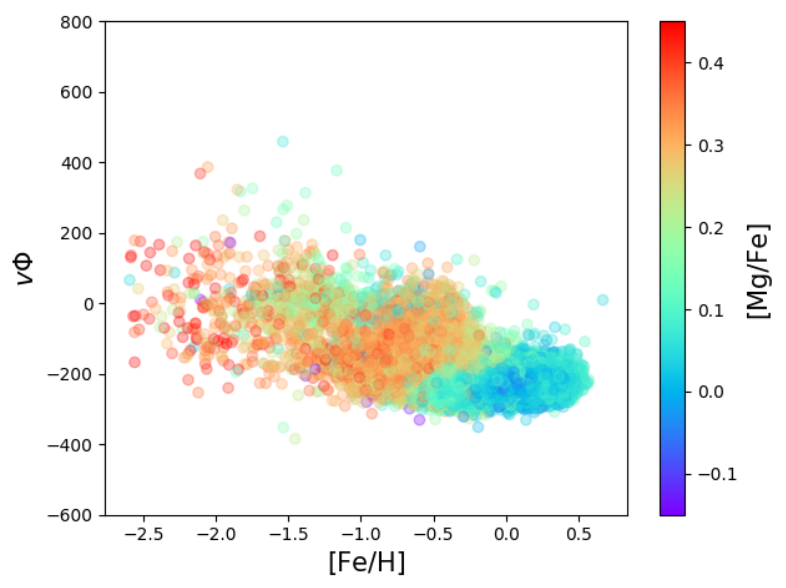

Fig. 11. Distribution of our sample of stars in the $[\mathrm{Fe} / \mathrm{H}]-v_{\Phi}$ plane The colours of each point represent their $[\mathrm{Mg} / \mathrm{Fe}]$ ratio as indicated by the colour bar to the right.

(1) stars with thick-disc kinematics can be found at metallicities as low as $[\mathrm{Fe} / \mathrm{H}] \sim-2$, thus at metallicities typical of the stellar halo; (2) stars with halo-like kinematics can also be found at metallicities typical of the thick disc and of the metalpoor thin disc as well (see, for example, Figs. 7-10). One of the consequences of this absence of well-defined borders is on the galactic chemo-kinematic relations at $[\mathrm{Fe} / \mathrm{H}] \geq-1$, that is at metallicities traditionally associated with disc populations. Minchev et al. (2014), for example, used RAVE and SEGUE data to derive these relations in an extended solar vicinity volume (about $1 \mathrm{kpc}$ in radius from the Sun position). Their derived velocity dispersions $-[\mathrm{Mg} / \mathrm{Fe}]$ relations, for different metallicity bins, show two main characteristics: (1) the velocity dispersions increase with $[\mathrm{Mg} / \mathrm{Fe}]$ for all metallicities $[\mathrm{Fe} / \mathrm{H}]>-0.4$, while the opposite trend is found for $[\mathrm{Fe} / \mathrm{H}]<-0.4$, where, at the highest magnesium abundances, the dispersions reach their minima; (2) the maxima reached in the velocity dispersion$[\mathrm{Mg} / \mathrm{Fe}]$ relations move to lower $[\mathrm{Mg} / \mathrm{Fe}]$ with decreasing metallicities.

In Minchev et al. (2014), these properties were interpreted as the signature of a significant merger in the early disc evolution, which would have increased the disc velocity dispersions to high values, and generated, at the same time, an inside-out migration of stars with cold kinematics. Both processes would have been responsible for causing the reversal in the velocity dispersions$[\mathrm{Mg} / \mathrm{Fe}]$ trends observed at $[\mathrm{Fe} / \mathrm{H}]<-0.4$. In more detail, in their interpretation, based on a detailed match to a chemodynamical model lacking, however, accreted populations, the metalpoor stars with high velocity dispersions and low $[\mathrm{Mg} / \mathrm{Fe}]$ ratios were considered, in-situ, as early disc stars heated by the interaction, while metal-poor stars with velocity dispersions as low as those of the metal-rich populations, and high $[\mathrm{Mg} / \mathrm{Fe}]$, were associated with in-situ stars migrated from the innermost regions of the early disc, as a result of the gravitational perturbation induced by the accreted satellite. Moreover, the displacement of the maximum of the velocity dispersion to lower $[\mathrm{Mg} / \mathrm{Fe}]$, the higher the metallicity, was suggested to be related to mergers of decreasing mass ratios that occurred during the Galaxy's lifetime.

The present analyses allow us to revisit the findings of Minchev et al. (2014) - see also Guiglion et al. (2015) for similar results - thanks to the exquisite details in kinematics and abundances of our sample (also $\sim 13$ times larger), and in particular permits us to address the role of accreted stars in shaping these relations, a role that has been underestimated in previous works.

Firstly, as explained in Sect. 3.1, it is the accreted population that drives the reversal of the velocity dispersions $-[\mathrm{Mg} / \mathrm{Fe}]$ relations at $[\mathrm{Fe} / \mathrm{H}]<-0.4$, where dispersions decrease with magnesium abundance. Because accreted stars describe a sequence of decreasing $[\mathrm{Mg} / \mathrm{Fe}]$ with increasing $[\mathrm{Fe} / \mathrm{H}]$, and are also characterised by the highest velocity dispersions, this naturally explains the displacement of the maximum of the velocity dispersions to higher $[\mathrm{Fe} / \mathrm{H}]$, as the $[\mathrm{Mg} / \mathrm{Fe}]$ decreases. Hence, this displacement is not related to a series of accretions, but is most probably due to the presence of stars from one accreted satellite only. Moreover, the reversal of the velocity dispersion- $[\mathrm{Mg} / \mathrm{Fe}]$ relation, found at $[\mathrm{Fe} / \mathrm{H}]<-0.4$, is due to the contamination by these accreted stars, which have very high velocities, and is not due to an intrinsic decrease of the velocity dispersion of the insitu population. In bins that are not contaminated by accreted stars $($ at $[\mathrm{Fe} / \mathrm{H}]>-0.5)$, the dispersions show no sign of a significant decrease in the highest abundance bins. At metallicities of -0.75 and -1 , low abundances $([\mathrm{Mg} / \mathrm{Fe}]<0.2)$ are contaminated by accreted stars, and show high dispersions, which decrease to lower values as the $[\mathrm{Mg} / \mathrm{Fe}]$ ratios increase, but simply reach levels of non-contaminated standard thick-disc stars, not the very low values found in Minchev et al. (2014). At even lower metallicities, even high- $[\mathrm{Mg} / \mathrm{Fe}]$ stars are contaminated by accreted stars, and show higher velocity dispersions, even if the decreasing trend remains visible in many cases. These trends are even clearer in Fig. 2, which shows that the velocity dispersion is uniform, or even rising, at the upper limit of the $[\mathrm{Fe} / \mathrm{H}]-[\mathrm{Mg} / \mathrm{Fe}]$ distribution, except in the region of the accreted sequence, in the lower right side of the distribution. To further probe the importance of the accreted component in shaping the galactic chemokinematic relations, and show the impact they have on the latter, in Fig. 17, we show the same chemo-kinematic relations already presented in Fig. 3. However, this time we only select stars with $[\mathrm{Fe} / \mathrm{H}]>-1$ and with $[\mathrm{Mg} / \mathrm{Fe}]>-0.26 \times[\mathrm{Fe} / \mathrm{H}]$, and stars with $[\mathrm{Mg} / \mathrm{Fe}] \leq-0.26 \times[\mathrm{Fe} / \mathrm{H}]$, but $[\mathrm{Fe} / \mathrm{H}]>-0.3$ (this is the same diagonal line adopted in Fig. 18, middle row). The selection appears severe, but it allows for a clean in-situ sample, not contaminated by the accreted sequence (see Fig. 17, top panel, blue points). The mean chemo-kinematic relations of this sample are also shown in Fig. 17 and demonstrate that when a clean in-situ sample is selected, no reversal is now found in any of the velocity dispersions $-[\mathrm{Mg} / \mathrm{Fe}]$ relations, thus further probing that this reversal is entirely generated by accreted stars.

Therefore, we cannot confirm the conclusions reached by Minchev et al. (2014). In particular, the shift of maxima in the velocity dispersion- $[\mathrm{Mg} / \mathrm{Fe}]$ relations to lower $[\mathrm{Mg} / \mathrm{Fe}]$ with decreasing metallicities interpreted by Minchev et al. (2014) as possibly being the consequence of successive accretions, is indeed most probably due to the accretion of a single satellite. Likewise, the decrease in velocity dispersions found by these authors at high- $[\mathrm{Mg} / \mathrm{Fe}]$ ratios, and interpreted as the signature of radial migration of kinematically colder stars from the inner to the outer disc, is also absent from our sample.

We conclude by emphasising that galactic chemo-kinematic relations, also in a metallicity regime often associated with the galactic disc $([\mathrm{Fe} / \mathrm{H}]>-1)$ are affected and reshaped by the accreted component(s), and that it is thus necessary to make selections as carefully as possible to be able to separate the contribution of these accreted stars from those of in-situ populations. The astrometric and spectroscopic quality of the data now available, and not achievable only few years ago, can now make these selections possible. 


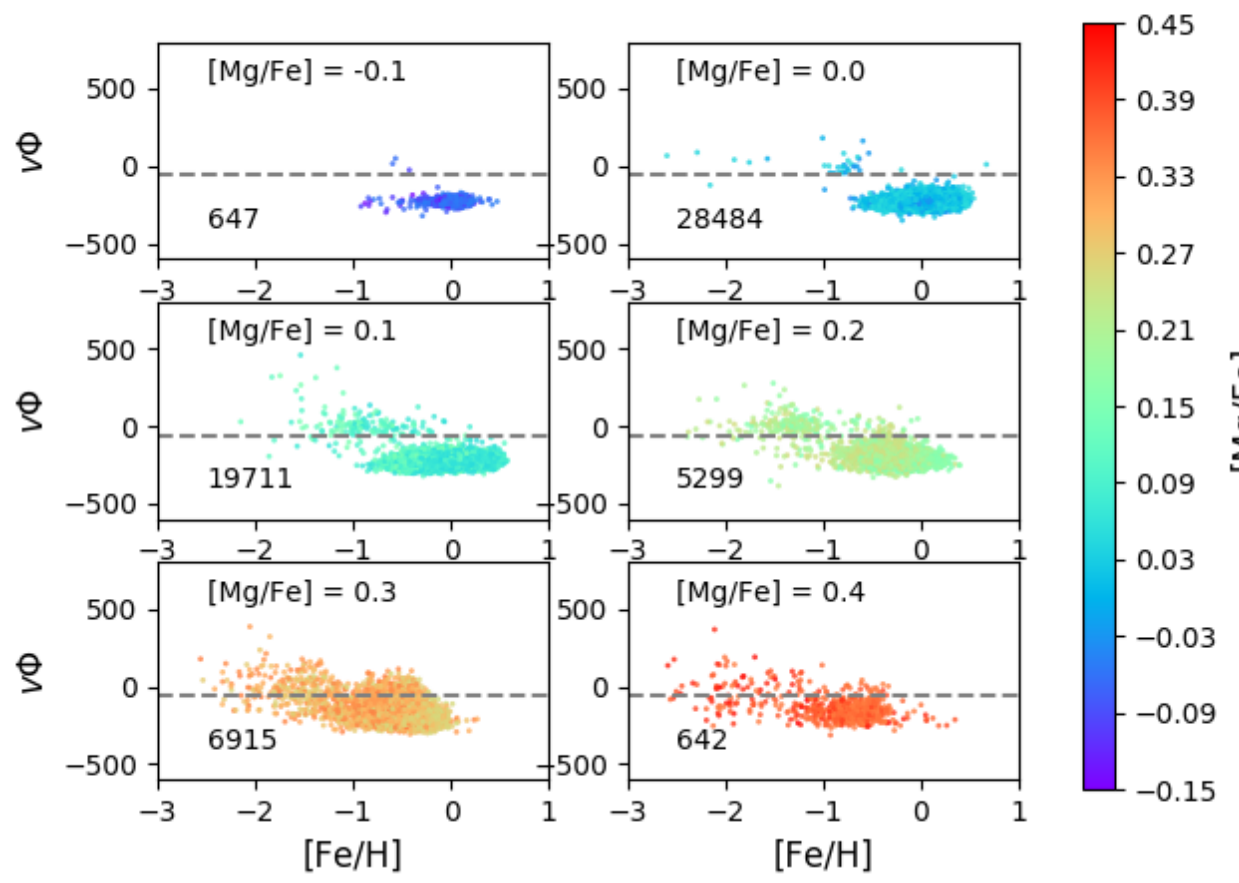

Fig. 12. Distribution of sample stars in $[\mathrm{Fe} / \mathrm{H}]-v_{\Phi}$ plane, for different intervals of $[\mathrm{Mg} / \mathrm{Fe}]$ abundances, as indicated in legends at the top of each panel. $[\mathrm{Mg} / \mathrm{Fe}]$ bins have been defined as in Fig. 4. The number of stars in each metallicity interval are also given in the lower-left corner of each panel. Colours of each point represent the $[\mathrm{Mg} / \mathrm{Fe}]$ ratio as indicated in the colour bar on the right-hand side of the figure.
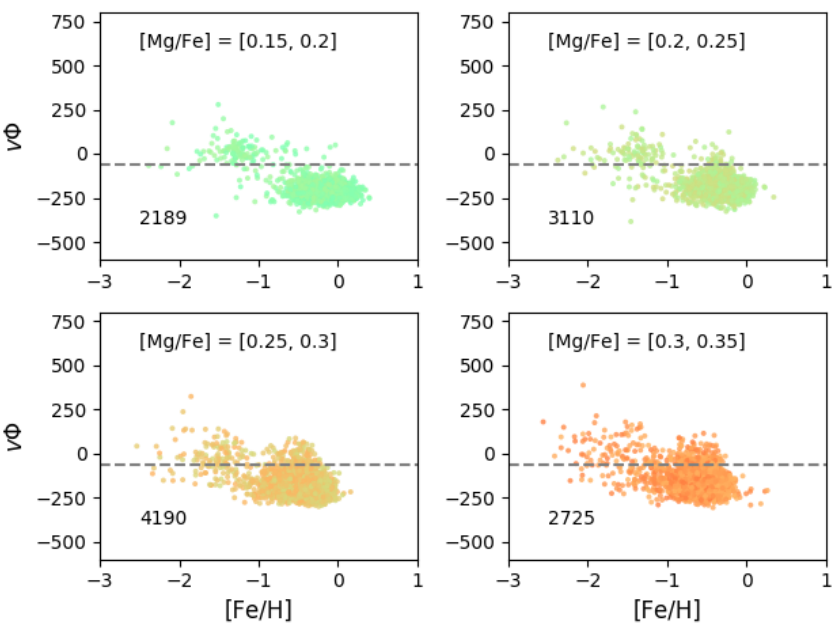

Fig. 13. Same as in Fig. 12, but for narrower intervals of fixed $[\mathrm{Mg} / \mathrm{Fe}]$ as indicated at the top of each panel, and now extending from $[\mathrm{Mg} / \mathrm{Fe}]=0.15$ to 0.35 . The colours of each point represent their $[\mathrm{Mg} / \mathrm{Fe}]$ ratios just as in Fig. 12. The number of stars in each $[\mathrm{Mg} / \mathrm{Fe}]$ bin is indicated to the lower left of each panel.

\subsection{The in-situ population below $[\mathrm{Fe} / \mathrm{H}]=-1$ is the galactic thick disc}

The halo population is, at first sight, an heterogeneous collection of stars. If the halo is defined as all stars with $[\mathrm{Fe} / \mathrm{H}]<-1$, our analysis shows that our sample, in this metallicity interval, is made for about $55-60 \%$ of accreted material, with weak prograde, null or retrograde rotation, and for the remaining 40-45\% of in-situ stars, with kinematics similar to that of thick disc stars in the metallicity range $[\mathrm{Fe} / \mathrm{H}]=[-1 .,-0.5]$. If the halo is defined on the basis of its kinematics, halo stars are found not only at $[\mathrm{Fe} / \mathrm{H}]<-1$, but also among stars with $[\mathrm{Fe} / \mathrm{H}]>-1$ : at $[\mathrm{Mg} / \mathrm{Fe}]$ typical of the thick disc, we find the in-situ heated disc that we will discuss further in the next section, and at $[\mathrm{Mg} / \mathrm{Fe}]$ typical of the metal-poor thin disc, we find the most metal-rich stars of the accreted population.
This complexity - and also ambiguity in the definition of the halo population is not new. The existence of metalpoor, $[\mathrm{Fe} / \mathrm{H}]<-1$, stars with thick-disc kinematics, usually referred to as the metal-weak thick disc, has been known for decades (Norris et al. 1985; Morrison et al. 1990, but see subsequent criticisms from Twarog \& Anthony-Twarog 1994, 1996). More recently, there has been tremendous effort expended to understand this thick-disc population (Chiba \& Beers 2000; Beers et al. 2002; Reddy \& Lambert 2008; Brown et al. 2008; Kordopatis et al. 2013; Hawkins et al. 2015; Li \& Zhao 2017; Hayes et al. 2018). Beers et al. (2002) estimated the fraction of stars in the metal-weak thick disc to be between $30 \%$ and $40 \%$ at $1.6<[\mathrm{Fe} / \mathrm{H}]<-1$, and suggested that this population could extend to metallicities as low as $[\mathrm{Fe} / \mathrm{H}] \lesssim-2$. These estimates correspond very closely to ours, since we find the fraction of stars with $[\mathrm{Fe} / \mathrm{H}]<-1$ and thick-disc kinematics to be $40-45 \%$ of the total fraction of stars at these metallicites, and with a contribution that decreases from $70 \%$ at $[\mathrm{Fe} / \mathrm{H}]-[\mathrm{Mg} / \mathrm{Fe}]=[[-1.5,-1],[0.25,0.35]]$, to $30 \%$ at $[\mathrm{Fe} / \mathrm{H}]-[\mathrm{Mg} / \mathrm{Fe}]=[[-2 .,-1.5],[0.25,0.35]]$ (see Sect. 3.2). Thus, our results confirm previous findings by Beers and collaborators that the fraction of metal-poor stars with thick-disc kinematics is significant, and it extends up to the lower limit of our sample, at $[\mathrm{Fe} / \mathrm{H}] \sim-2$. The recent finding by Sestito et al. (2019) of several very low-metallicity stars with disc kinematics is a first step towards extending these estimates to the very metal-poor stars.

Interestingly, the fraction of metal-poor stars with thick-disc kinematics also corresponds very closely to early estimates by Sommer-Larsen \& Zhen (1990), who found a metal-poor halo consisting of two components. One component is highly flattened, confined in the inner parts of the Galaxy, and contributes $\approx 40 \%$ of the density of metal-poor stars near the Sun. For all these reasons, we conclude that stars with thick disc kinematics below and above $[\mathrm{Fe} / \mathrm{H}]=-1$ constitute the same population, commonly referred to as the thick disc, thus strengthening previous findings of a continuity between the thick disc and its metalpoor extension (Hawkins et al. 2015; Hayes et al. 2018). Among the thick-disc population, stars with $[\mathrm{Fe} / \mathrm{H}] \leq-0.3$ have been 


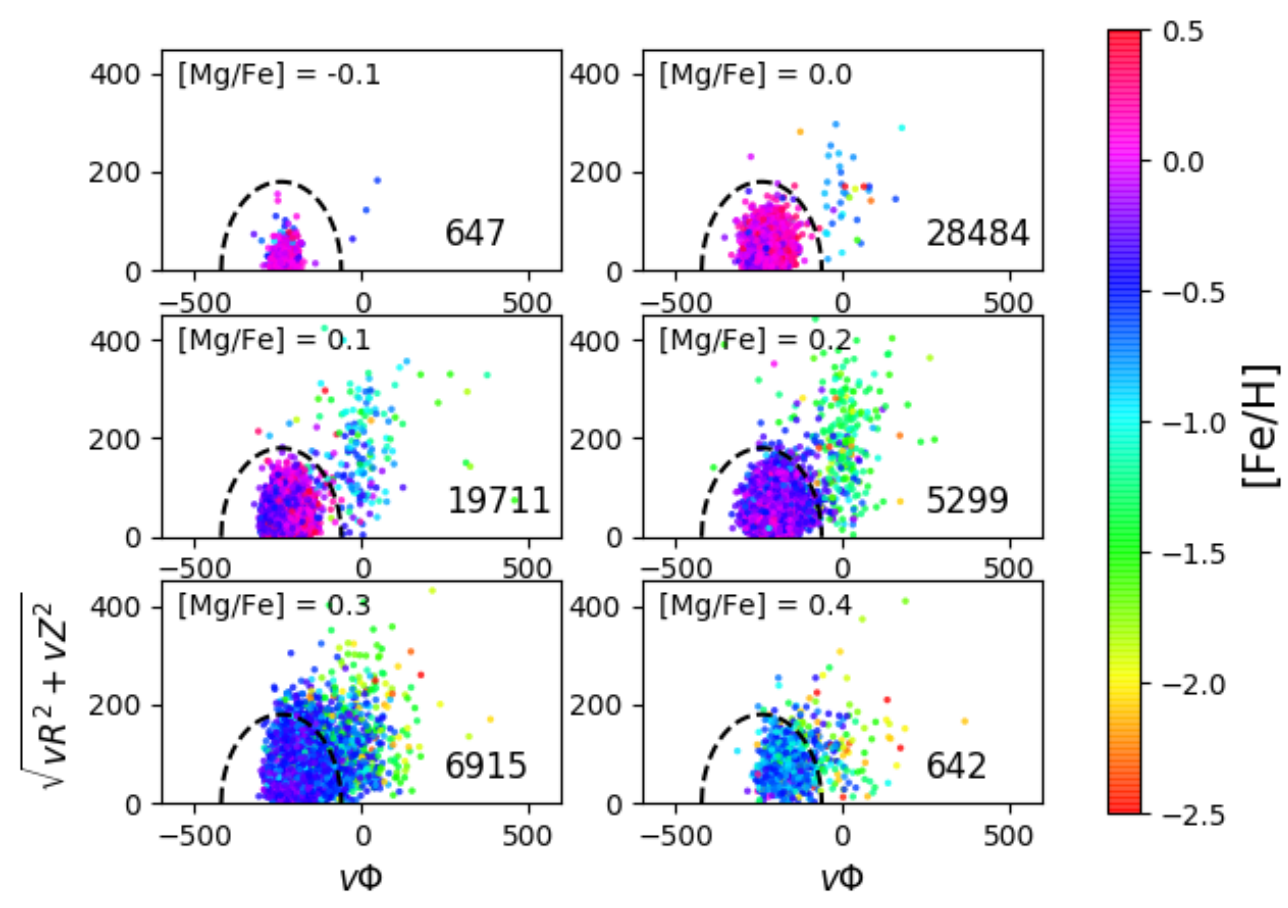

Fig. 14. Distribution of sample stars in Toomre diagram, for different intervals of $[\mathrm{Mg} / \mathrm{Fe}]$ abundances, as indicated at top left of each panel. $[\mathrm{Mg} / \mathrm{Fe}]$ bins have been defined as in Fig. 4. The number of stars in each metallicity interval is given in the lower-right corner of each panel. The colour of each point indicates its $[\mathrm{Fe} / \mathrm{H}]$ ratio as given in the colour bar shown to the right of the figure.

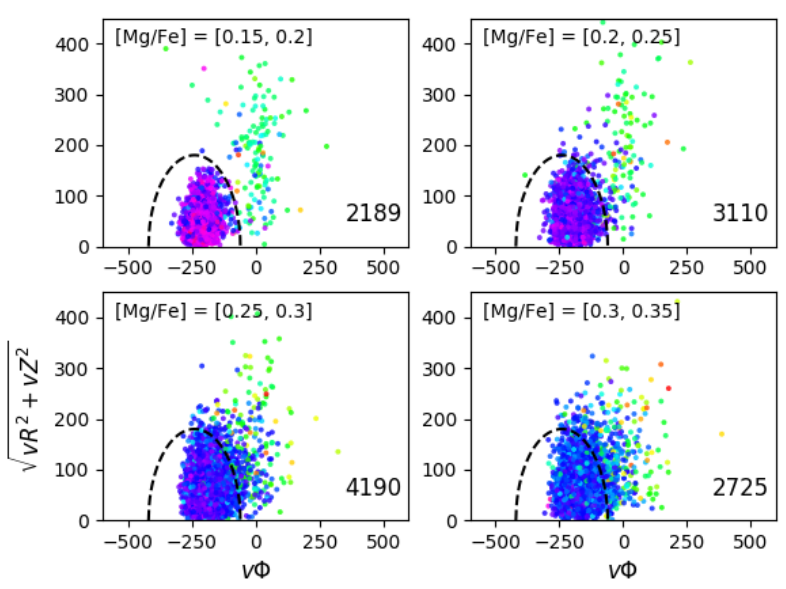

Fig. 15. Same as in Fig. 14, but for narrower $[\mathrm{Mg} / \mathrm{Fe}]$ intervals, extending from $[\mathrm{Mg} / \mathrm{Fe}]=0.15$ to 0.35 . Colours of each point represent their $[\mathrm{Fe} / \mathrm{H}]$ ratios, as in Fig. 14. The number of stars in each $[\mathrm{Mg} / \mathrm{Fe}]$ bin is indicated at the lower right of each panel.

significantly heated by the accretion of the satellite whose debris constitutes the dominant halo population, at $[\mathrm{Fe} / \mathrm{H}]<-1$ and at few kpc from the Sun.

The second component that we find at $[\mathrm{Fe} / \mathrm{H}]<-1$ belongs to the accreted population discovered by Nissen \& Schuster (2010), and - in the metallicity range explored in this study - this component appears to be the dominant one, constituting about $55-60 \%$ of the total metal-poor populations at few kpc from the Sun. Currently, we find no evidence in our study for a third, non-rotating, in-situ component: the non-rotating inner halo by Carollo et al. $(2007,2010)$ is made, in our interpretation, only of stars belonging to the accreted population and of the low velocity tail of the thick disc at these metallicities. As already discussed by Haywood et al. (2018), the stars in the halo at metallicities below $[\mathrm{Fe} / \mathrm{H}]=-1$ are dominated by stars accreted from the merging galaxy. The only other significant component that we find at these metallicities is the metal-poor thick disc, partly heated by the accretion to kinematics typical of halo stars, and

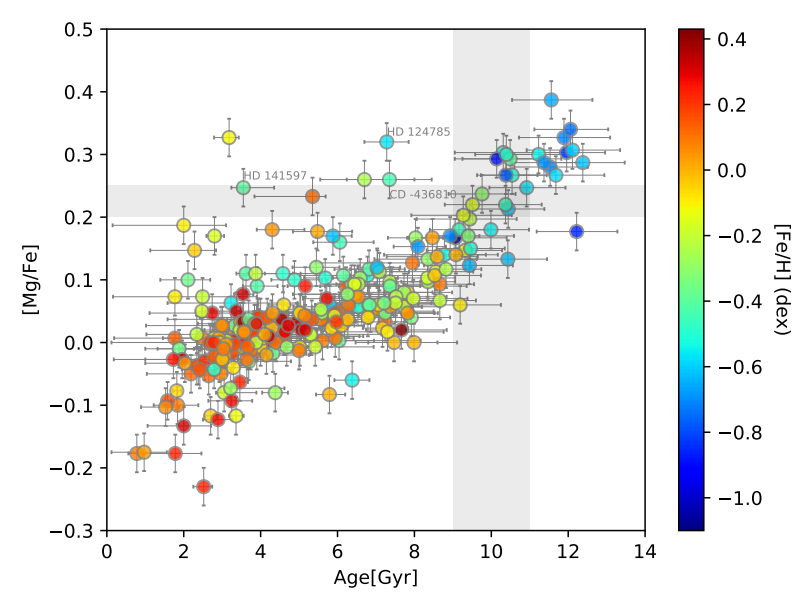

Fig. 16. Age- $[\mathrm{Mg} / \mathrm{Fe}]$ distribution for stars in Adibekyan sample for which we could determine ages and that have $M v<4.5$. The horizontal grey band corresponds to the $\mathrm{Mg}$ abundance where kinematically heated stars appear. It corresponds to an age range from nine to $11 \mathrm{Gyr}$. Three stars are confirmed to be "young", alpha-rich objects, their name is indicated in the figure (see text for details).

thus at least partially overlapping with the population of accreted stars. The existence of in-situ original non-rotating halo stars, that is to say, the collapsed halo - once accreted stars and metalpoor thick-disc stars heated by the interaction are removed remains to be demonstrated.

\subsection{The kinematically defined halo is predominantly made of in-situ, metal-rich, $[\mathrm{Fe} / \mathrm{H}]>-1$, disc stars heated by mergers}

In Jean-Baptiste et al. (2017), we analysed N-body simulations of the accretion of one or several satellites onto a Milky Way-type galaxy to investigate the possibility of discriminating the origin of stars - in-situ or accreted - on the basis of their kinematics alone, as suggested by other studies (see, for example Helmi \& de Zeeuw 2000; Gómez et al. 2010). One of the main conclusions 

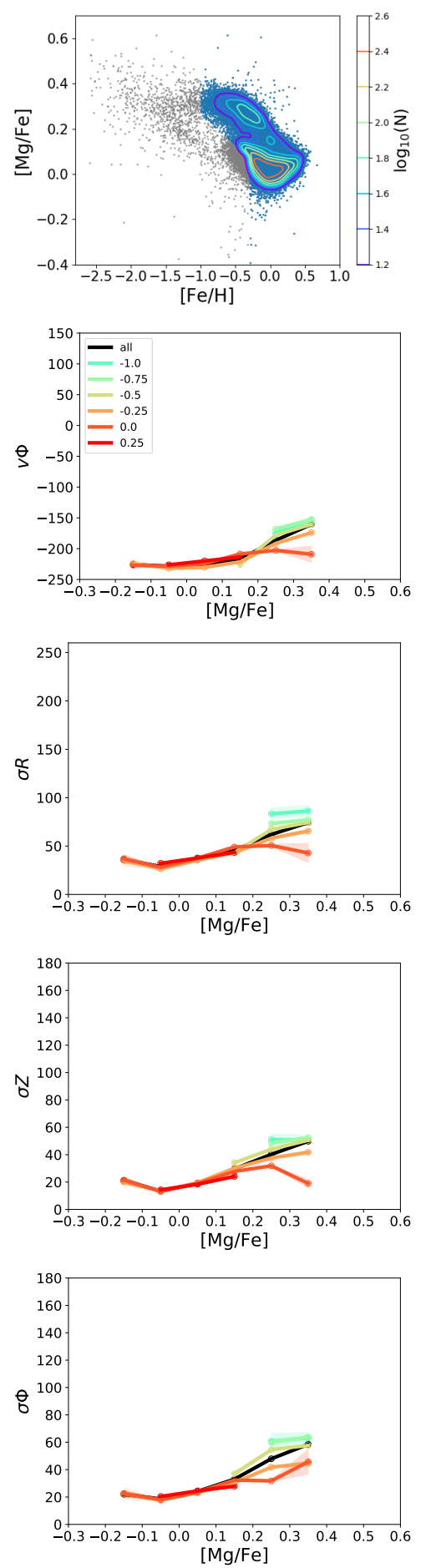

Fig. 17. Top panel: distribution of stars of in-situ sample (blue points) in $[\mathrm{Fe} / \mathrm{H}]-[\mathrm{Mg} / \mathrm{Fe}]$ plane. Coloured lines correspond to isodensity contours, in logarithmic scale, as indicated in the colour bar to the righthand side of the panel. For comparison, all stars of the main sample studied in this paper, and shown in Fig. 2, are shown by grey points. From the second to the bottom panel: mean azimuthal velocity, radial, vertical and azimuthal velocity dispersion of stars of in-situ sample, as a function of their $[\mathrm{Mg} / \mathrm{Fe}]$ ratio. In each panel, the relations are given for bins in $[\mathrm{Fe} / \mathrm{H}]$, as indicated in the legend in the top panel. The black curves show the corresponding relation, for the total sample not binned in $[\mathrm{Fe} / \mathrm{H}]$. The $1 \sigma$ uncertainty in each relation (coloured, shaded regions) is estimated through 1000 bootstrapped realisations. In all panels, only bins containing more than ten stars are shown. In all plots, we adopted the same $y$-axis limits, as adopted in Fig. 3, to facilitate the comparison. of Jean-Baptiste et al. was that distinguishing accreted and in-situ populations on the basis of kinematics alone would be virtually impossible without detailed chemical abundances. They reached this conclusion because the populations have overlapping kinematics. In their simulations, indeed, in solar volumes of few kpc in size, the dominant halo component - defined as stars with hot kinematics - was found to be made of in-situ material (see also Font et al. 2011; McCarthy et al. 2012). Figures 14-17 already indicate that an in-situ halo, made of stars with metallicities typical of the galactic thick disc with $[\mathrm{Fe} / \mathrm{H}]>-1$ are present in the data. That stars with halo kinematics and $[\mathrm{Fe} / \mathrm{H}]>-1$ exist in the Galaxy is not a new finding, as it has been discussed in a number of previous studies (see, for example Nissen \& Schuster 2010, 2011; Schuster et al. 2012; Jackson-Jones et al. 2014; Bonaca et al. 2017; Fernández-Alvar et al. 2019b; Gaia Collaboration et al. 2018b). Here, we want to take a step further, by quantifying how significant this population is to the total halo population at few kpc from the Sun.

Figure 18 (top-left panel) shows the Toomre diagram of all stars in our sample with halo kinematics, meaning all stars in the sample with $\sqrt{\left(v_{\Phi}-v_{\mathrm{LSR}}\right)^{2}+v_{R}^{2}+v_{Z}^{2}}>180 \mathrm{~km} \mathrm{~s}^{-1}$. To isolate the contribution of thick-disc stars with $[\mathrm{Fe} / \mathrm{H}]>-1$, we have drawn an oblique line in the $[\mathrm{Fe} / \mathrm{H}]-[\mathrm{Mg} / \mathrm{Fe}]$ plane that intercepts the thick disc at $[\mathrm{Fe} / \mathrm{H}]=-1$ and $[\mathrm{Mg} / \mathrm{Fe}]=0.3$, and which separates the low- $\alpha$ portion of the accreted sequence from the thin and thick discs relatively well (middle-left panel). Among stars with thick-disc abundances and $[\mathrm{Fe} / \mathrm{H}]>-1$, 830 stars have halo kinematics. For comparison, the number of stars of the accreted and in-situ sequence to the left of the diagonal line, with halo kinematics, is 717 . Our sample thus indicates that at few $\mathrm{kpc}$ from the Sun, the majority of the kinematically-defined halo is made of stars with $[\mathrm{Fe} / \mathrm{H}]>-1$ and $[\mathrm{Mg} / \mathrm{Fe}]$-abundances of the early thick disc, heated by the interaction. This is a very nice confirmation of the predictions by Jean-Baptiste et al. (2017), based on the analysis of N-body models, which suggested that in volumes of few kpc around the Sun, the kinematically-defined halo should be dominated by in-situ disc stars heated by one or several merger(s).

These heated-disc stars do not only constitute the majority of the kinematically-defined halo in the region under study, but also a non-negligible fraction of $\alpha$-abundant stars at $[\mathrm{Fe} / \mathrm{H}]>-1$. Figure 18 (bottom-left panel) indeed shows the fraction of stars with halo kinematics, in various regions of the $[\mathrm{Fe} / \mathrm{H}]-[\mathrm{Mg} / \mathrm{Fe}]$ plane. At high $[\mathrm{Mg} / \mathrm{Fe}]$ and $-1<[\mathrm{Fe} / \mathrm{H}]<-0.3$, thus in a range typical of the thick disc, the fraction of halo stars can be as high as $20 \%$. This implies that any selection of thick-disc stars made only on the basis of chemical abundances could lead to a significant contamination by stars with significantly hotter kinematics, on halo-like orbits. Of course the above fractions change if we change the limiting velocities discriminating disc stars from halo stars. If, for example, we adopt a kinematic definition for the halo as all stars with $\sqrt{\left(v_{\Phi}-v_{\mathrm{LSR}}\right)^{2}+v_{R}^{2}+v_{Z}^{2}}>220 \mathrm{~km} \mathrm{~s}^{-1}$, the fractional contribution of the heated thick disc diminishes, and, conversely, the contribution of stars on the left of the diagonal line increases (top, middle and bottom-right panels). With this selection, the fraction of metal-rich thick-disc stars with halo kinematics indeed decreases to $38 \%$ of the total sample of kinematically-selected halo stars. Still, such a selection is not able to completely eliminate the contamination of these stars to the canonical thick-disc population, and indeed, as shown on the bottom-right panel of this Figure, the fraction of halo stars in the region of the $[\mathrm{Fe} / \mathrm{H}]-[\mathrm{Mg} / \mathrm{Fe}]$ plane where thick-disc stars with $-1<[\mathrm{Fe} / \mathrm{H}]<-0.3$ are located can be as high as $10 \%$. 

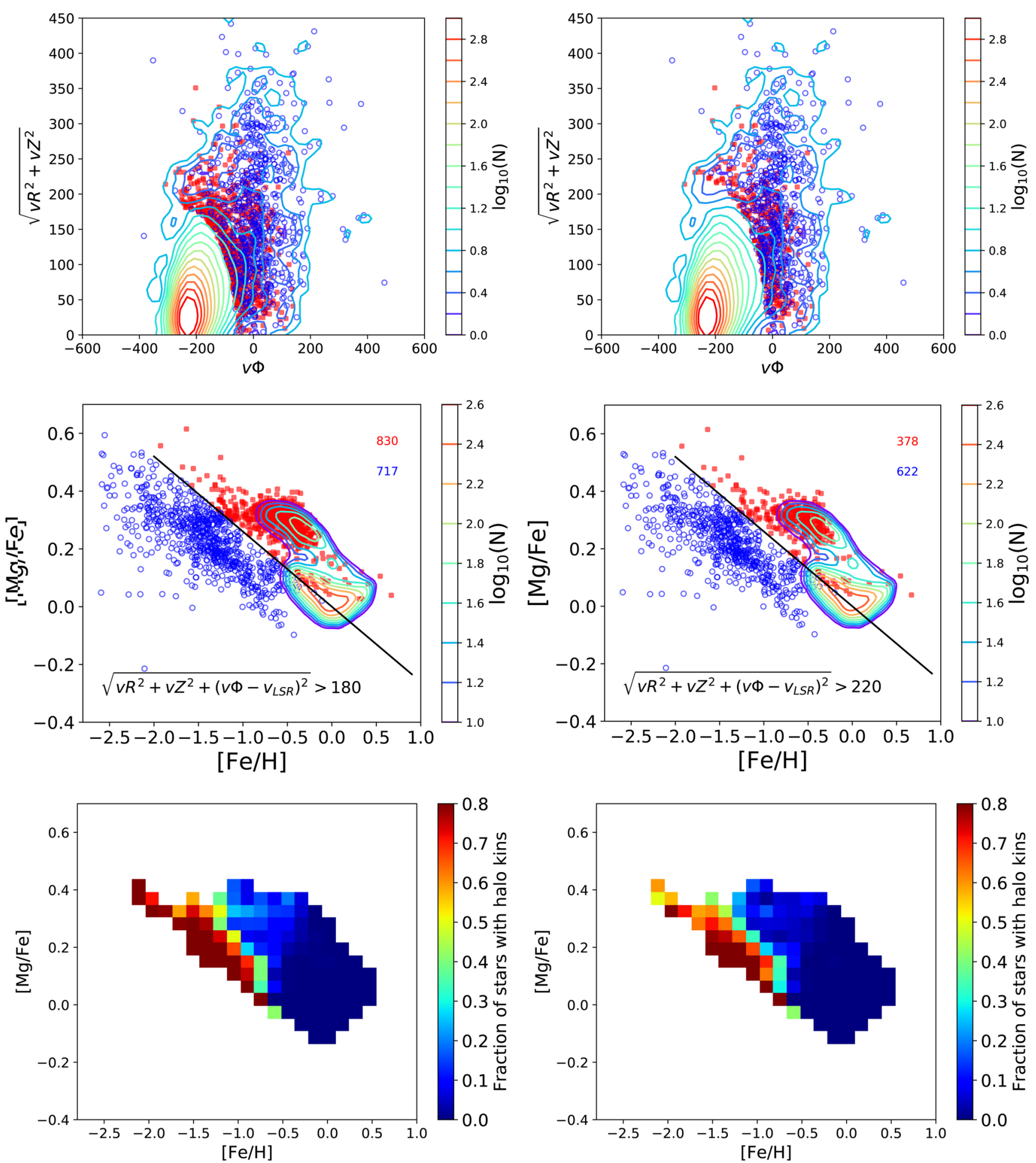

Fig. 18. First row: Toomre diagram of all stars in our sample with halo-like kinematics. The distributions in the right and left panels differ for the threshold adopted in defining halo stars, as reported in the middle row. Red points indicate metal-rich thick-disc stars, blue points indicate accreted and metal-poor thick-disc stars (see middle row and text for their definition). Coloured lines correspond to isodensity contours for the whole sample analysed in this paper. Middle row: distribution, in the $[\mathrm{Fe} / \mathrm{H}]-[\mathrm{Mg} / \mathrm{Fe}]$ plane, of stars of sample with halo-like kinematics. The diagonal line separates the metal-rich thick-disc sequence, in red on the right, from the accreted and metal-poor thick disc, in blue on the left. The distributions in the right and left panels differ for the threshold adopted in defining halo stars, as reported in the plots. The number of stars on the left and right of the diagonal lines are given in both panels, in blue and red, respectively. Coloured lines correspond to isodensity contours, as defined in Fig. 2. Bottom row: fraction of stars with halo-like kinematics, in $[\mathrm{Fe} / \mathrm{H}]-[\mathrm{Mg} / \mathrm{Fe}]$ plane, for two different definitions of halo, adopted for left panels. In both plots, the fraction is normalised to the total number of stars in a given pixel.

The significant contribution of the metal-rich thick-disc population to the kinematically-defined halo is also shown in Fig. 19, where the metallicity distribution of kinematically-defined halo stars is shown for the two limiting velocities discriminating disc stars from halo stars, as described above. Even when adopting the restrictive threshold of $\sqrt{\left(v_{\Phi}-v_{\mathrm{LSR}}\right)^{2}+v_{R}^{2}+v_{Z}^{2}}>220 \mathrm{~km} \mathrm{~s}^{-1}$, 

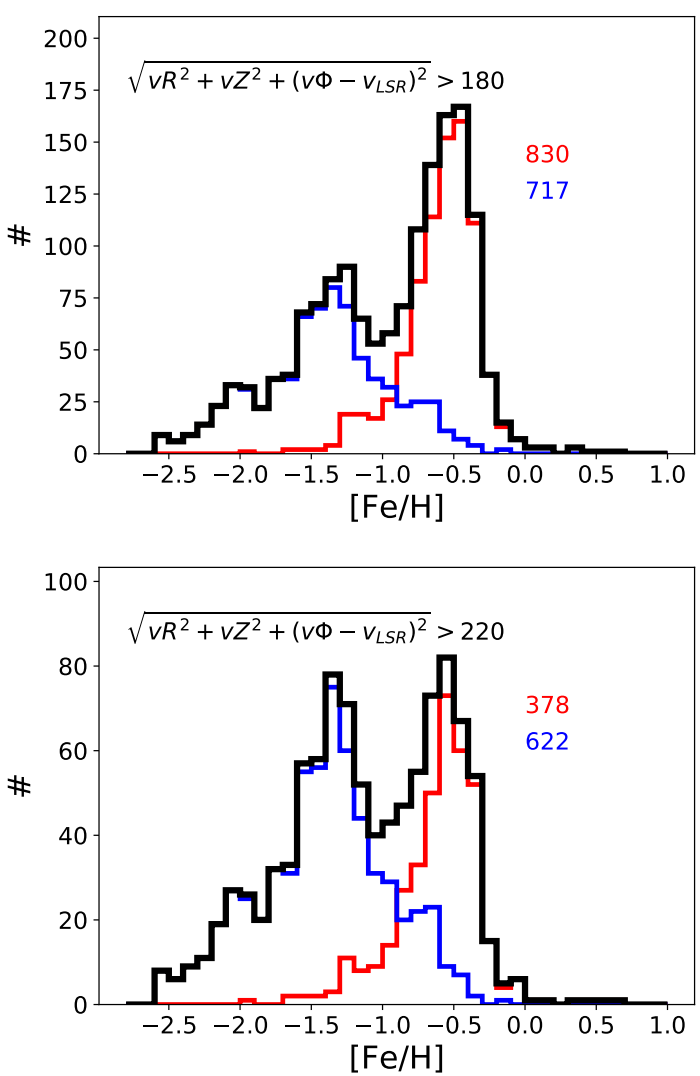

Fig. 19. Metallicity distribution of stars in sample with halo-like kinematics (black curve). The contribution of metal-rich thick-disc stars, and of accreted and metal-poor thick disc stars to the total distribution is shown, respectively, by the red and blue curves. Two different limiting velocities for discriminating halo stars is adopted in the top and bottom panels, as reported in the legends.

the peak associated with the metal-rich thick-disc population clearly stands out. Interestingly, this metallicity distribution is strongly reminiscent of the distribution of metallicity versus distance from the plane found by Ibata et al. (2017) and of that found by Gallart et al. (2019).

These metal-rich, heated-disc stars have a velocity ellipsoid of $\left(\sigma_{\mathrm{R}}, \sigma_{\Phi}, \sigma_{\mathrm{Z}}\right)=(124 \pm 3 ., 72 . \pm 2 ., 72 . \pm 2.) \mathrm{km} \mathrm{s}^{-1}$, when a threshold of $180 \mathrm{~km} \mathrm{~s}^{-1}$ is adopted in defining halo stars, and $\left(\sigma_{\mathrm{R}}, \sigma_{\Phi}, \sigma_{\mathrm{Z}}\right)=(134 \pm 5 ., 70 . \pm 3 ., 81 . \pm 3.) \mathrm{km} \mathrm{s}^{-1}$ for a threshold of $220 \mathrm{~km} \mathrm{~s}^{-1}$.

As seen in Sect. 2, our sample is still relatively local and biased against the inner populations of the Galaxy, because it lacks stars in the fourth quadrant, and because most of its stars are beyond the solar circle. However, we know from previous studies that the $\alpha$-abundant thick disc is massive (Snaith et al. $2014,2015)$ and mostly confined to the inner-galactic regions (Bensby et al. 2011; Bovy et al. 2012, 2016; Cheng et al. 2012), meaning inside the solar circle. Under the hypothesis that the fraction of stars with halo-like kinematics, but thick-disc abundances, as found here, can be representative of the whole inner Galaxy, this would imply that the mass of thick-disc stars at $[\mathrm{Fe} / \mathrm{H}]>-1$ with halo-like kinematics would be significant, being between $10 \%-20 \%$ of the total thick-disc mass, which is between $2-5 \times 10^{9} M_{\odot}{ }^{6}$. This would make this component by far the dominant halo component in the inner Galaxy, at $R \leq 8-10 \mathrm{kpc}$. These estimates must, of course, be taken with

\footnotetext{
6 For a total stellar mass of the Galaxy of $5 \times 10^{10} M_{\odot}$.
}

caution, because they are derived by extrapolating local fractions. However, they do suggest that the in-situ heated disc may be the dominant halo component not only in the region under study, but in the inner Galaxy as well.

\subsection{Heating versus cooling scenario}

So far, we have interpreted our findings as the signature of a major heating event in the Galaxy. Here, we want to briefly discuss why we favour this interpretation over the one where the in-situ population at retrograde or null rotation (i.e. the plume found in Figs. 12 and 13) is made of stars formed from a cooling gaseous disc. While the distinction between a heated disc and a cooling one could be difficult to establish, we believe that some conclusions can be derived on the basis of the metallicities of stars that make up the plume. The plume is visible up to $[\mathrm{Fe} / \mathrm{H}]$ as high as -0.3 , where the vast majority of stars already have disc kinematics. If the cooling of the gaseous disc left signatures in the stellar populations, we expect them to be found at $[\mathrm{Fe} / \mathrm{H}]$, which pre-dates the disc formation, but not at metallicities where the disc is already fully formed. Conversely, in the case of a heating scenario, we expect exactly the signature we observe: a fraction of disc stars - with the same chemical abundances - to have halo (prograde and retrograde) kinematics. As discussed in the previous sections, indeed, in this scenario, an initial (before the merger(s)) same in-situ disc population would end up (after the merger(s)) having mostly disc-kinematics, while a fraction of it would be heated up to the halo. The prediction is thus to have the same abundances, but different kinematics, which is exactly what we see in the data. Moreover, the prediction of a heating scenario is that part of the heated halo stars should have kinematic properties overlapping with those of the accreted retrograde material (Jean-Baptiste et al. 2017), which is what we find: stars with in-situ chemistry and retrograde motions. The presence of retrograde motions in the plume, in particular, seems to favour the heating scenario, because in a cooling disc, while an increase of the angular momentum is expected with time, it is difficult to explain how to maintain part of a cooling gaseous disc in counter-rotation, because of its dissipative nature.

\subsection{The epoch of the last significant accretion and the question of an in-situ stellar halo}

The epoch of accretion that we measure is in agreement with what has been inferred in various studies (Belokurov et al. 2018; Mackereth et al. 2019; Helmi et al. 2018; Gallart et al. 2019). It is also consistent with the epoch of last significant merging experienced by the Galaxy, derived by Kruijssen et al. (2019) based on the analysis of the globular cluster population. The difference, however, is that we find the fossil record left by the accretion in the kinematic properties of the disc at $[\mathrm{Fe} / \mathrm{H}] \leq-0.3$ and $[\mathrm{Mg} / \mathrm{Fe}] \geq 0.2$, corresponding to nine to $11 \mathrm{Gyr}$ ago. This is in particular in agreement with the inference made by Belokurov et al. (2018) that the accretion must have occurred at a time when the disc was already substantially massive, in order to produce the velocity anisotropy that is observed. This is also in agreement with the results found by Mackereth et al. (2019), who show, analysing the Eagle simulations, that material on high eccentricities cannot have been accreted too early. Hence, an accretion occurring nine to $11 \mathrm{Gyr}$ ago falls at the right time, that is when the formation of the thick disc was ongoing or approaching near completion, reaching a stellar mass between 1 and $2.10^{10} M_{\odot}$ (see Snaith et al. 2015). It also corresponds to a time when the 
star formation in the Galaxy was particularly intense (see Snaith et al. 2014; Lehnert et al. 2014).

As anticipated in Haywood et al. (2018), the accretion event was responsible for heating the disc, and even pushing a fraction of the thick-disc stars on counter-rotating orbits and orbits on higher eccentricities, populating the kinematically defined halo. With the results presented here, the question of the existence of an in-situ halo, other than the heated early disc, as raised already in Haywood et al. (2018), is even more acute.

Even on larger scales, the evidence of an in-situ halo other than the heated early Milky Way disc is becoming weaker by the day. For instance, the recent work by Iorio \& Belokurov (2019) shows that the distribution of RR Lyrae over the entire Galaxy (after being cleaned of known structures) has kinematic properties suggesting a strong anisotropy compatible with accreted material, the so-called "Gaia Sausage", in their study. On the large scale, the Gaia Sausage stars would be distributed in a triaxial structure whose major axis would be aligned at $70^{\circ}$ with the Sun-galactic centre direction. According to Simion et al. (2019), the Hercules-Aquila cloud and the Virgo Over-Density, possibly aligned with a major axis of the Gaia Sausage, could be part of the same accretion event. Iorio \& Belokurov (2019) conclude from their analysis that the bulk of the triaxial structure made by RR Lyrae stars must have been left by this accretion event.

An entirely new and unexpected result also supports the view that a spherical isotropic in-situ halo is a very small component of the Milky Way (much less massive than the $\sim 1 \%$ of stars that were up to now viewed as the contribution of the galactic halo). The discovery of stars with disc orbits at very low metallicities $([\mathrm{Fe} / \mathrm{H}]<-4$, see Sestito et al. 2019) suggests that the dissipative collapse that led to the formation of the thick disc was probably extremely rapid (within $10^{8}$ years?), leaving very little time to build a spherical, in-situ halo. This argument, which is reminiscent of the rapid collapse advocated by Eggen et al. (1962), suggests that the Galaxy may have started to form stars in a disc configuration very soon after the Big-Bang, leaving only a very short amount of time to form stars in a more spheroidal configuration.

\section{Conclusions}

By coupling astrometric data from Gaia DR2, with elemental abundances from APOGEE DR14, we continue our study on the nature of the galactic halo at few kpc from the Sun, started in Haywood et al. (2018). Here, in particular, we characterise the kinematics and chemistry of in-situ and accreted populations in the Galaxy up to $[\mathrm{Fe} / \mathrm{H}] \sim-2$. Our results can be summarised as follows.

In the $[\mathrm{Fe} / \mathrm{H}]-[\mathrm{Mg} / \mathrm{Fe}]$ plane, the chemical sequence of accreted stars - firstly identified by Nissen \& Schuster (2010), and later shown by Haywood et al. (2018) to be the dominant component among stars with $[\mathrm{Fe} / \mathrm{H}]<-1-$ remarkably appears as a distinct sequence in terms of its kinematics, being characterised by a mean null or retrograde motion and significantly higher velocity dispersions than those of disc stars and halo stars with higher $[\mathrm{Mg} / \mathrm{Fe}]$ ratios, but the same metallicities.

Accreted stars appear to significantly impact the galactic chemo-kinematic relations, not only at $[\mathrm{Fe} / \mathrm{H}] \leq-1$, but also at metallicities typical of the thick and metal-poor thin discs. In this context, we can revisit the finding of Minchev et al. (2014) (see also Guiglion et al. 2015) and conclude that: (1) the inversion they found in velocity dispersions $-[\mathrm{Mg} / \mathrm{Fe}]$ relations at $[\mathrm{Fe} / \mathrm{H}]<-0.4-$ where stars with the lowest $[\mathrm{Mg} / \mathrm{Fe}]$ ratios also have the highest velocity dispersions - is exclusively driven by the accreted population, and not by in-situ disc stars heated by the accretion. In-situ disc stars heated by the interaction are indeed characterised by high $[\mathrm{Mg} / \mathrm{Fe}]$ ratios, and not low $[\mathrm{Mg} / \mathrm{Fe}]$, as expected in their scenario; (2) there is no sign of a drop in the velocity dispersion of stars at the high $[\mathrm{Mg} / \mathrm{Fe}]$ end, indicative, in the interpretation of Minchev et al. (2014), of a significant radial migration from the inner to the outer disc.

We show that stars with thick disc kinematics are present up to the low $[\mathrm{Fe} / \mathrm{H}]$ end of our sample $([\mathrm{Fe} / \mathrm{H}] \sim-2)$, and that they constitute about $40 \%$ of all stars with $[\mathrm{Fe} / \mathrm{H}]<-1$. This estimate corresponds very well to the findings of Beers et al. (2002). The remaining $60 \%$ is made of accreted stars, which confirming the finding in Haywood et al. (2018) - represent the majority of stars at these metallicities.

While stars at $[\mathrm{Fe} / \mathrm{H}]<-1$ are a mix of accreted and thickdisc stars, when the halo is defined on the basis of the kinematics of its stars, the majority is made, at few kpc from the Sun, of metal-rich (i.e. $[\mathrm{Fe} / \mathrm{H}]>-1)$ stars heated by the interaction. These stars have the same chemical composition of the thick disc, but hotter kinematics. Their existence in large proportions in the inner Galaxy has been predicted by a number of N-body models, (Zolotov et al. 2010; Purcell et al. 2010; Font et al. 2011; Qu et al. 2011a; McCarthy et al. 2012; Jean-Baptiste et al. 2017) and here we show that they indeed constitute a significant kinematically "hot" component in the volume under study.

Based on these findings, we tentatively estimate this earlydisc component heated by the interaction to have a total stellar mass of about $2-5 \times 10^{9} M_{\odot}$ in the inner Galaxy, meaning, inside $R=8-10 \mathrm{kpc}$. If these estimates are confirmed in future studies, this would make this component by far the dominant halo component of the inner Galaxy.

By constraining the metallicity and $[\mathrm{Mg} / \mathrm{Fe}]$ ratio of these disc stars heated to halo kinematics, we can now date the major accretion event reported in a number of studies (Nissen \& Schuster 2010, 2011; Schuster et al. 2012; Ramírez et al. 2012; Hawkins et al. 2015; Hayes et al. 2018; Belokurov et al. 2018; Haywood et al. 2018; Iorio \& Belokurov 2019; Helmi et al. 2018; Mackereth et al. 2019; Gallart et al. 2019) to have occurred between nine and $11 \mathrm{Gyr}$ ago. To our knowledge, this is the first time this accretion is dated on the basis of the kinematic imprints it left on disc stars present in the Galaxy at the time it occurred.

The picture that emerges from our study, and that confirms the earliest conclusions in Haywood et al. (2018), is that an in-situ halo, other than the heated thick disc, possibly does not exist in our Galaxy, or it represents much less than some few percent of stars, as usually reported in the literature. The only in-situ population that we find in great proportions both among the kinematically defined and the chemically defined halo stars is the thick disc, which is the early disc of the Galaxy heated to hot kinematics. This population indeed constitutes a significant or dominant contributor to the inner halo, and cannot be neglected in all discussions about its origins. As for a distinctive in-situ halo population, if it exists, it is further beyond our reach.

Acknowledgements. The authors wish to thank P. Bonifacio, E. Caffau, D. Kruijssen and I. Minchev for valuable discussions and remarks on this work. The authors are grateful to the referee, for their very constructive report which much improved the manuscript. This work has been supported by the ANR (Agence Nationale de la Recherche) through the MOD4Gaia project (ANR-15-CE31-0007, P.I.: P. Di Matteo). SK was supported by the Russian Science Foundation, project no. 19-72-20089. This work has made use of data from the European Space Agency (ESA) mission Gaia (https://www . cosmos.esa.int/gaia), processed by the Gaia Data Processing and Analysis Consortium (DPAC, https://www. cosmos.esa.int/web/gaia/dpac/ consortium). Funding for the DPAC has been provided by national institutions, in particular the institutions participating in the Gaia Multilateral Agreement. 
This research has made use of the SIMBAD database, operated at CDS, Strasbourg, France. Funding for the Sloan Digital Sky Survey IV has been provided by the Alfred P. Sloan Foundation, the U.S. Department of Energy Office of Science, and the Participating Institutions. SDSS-IV acknowledges support and resources from the Center for High-Performance Computing at the University of Utah. The SDSS web site is www.sdss.org. SDSS-IV is managed by the Astrophysical Research Consortium for the Participating Institutions of the SDSS Collaboration including the Brazilian Participation Group, the Carnegie Institution for Science, Carnegie Mellon University, the Chilean Participation Group, the French Participation Group, Harvard-Smithsonian Center for Astrophysics, Instituto de Astrofísica de Canarias, The Johns Hopkins University, Kavli Institute for the Physics and Mathematics of the Universe (IPMU) / University of Tokyo, Lawrence Berkeley National Laboratory, Leibniz Institut für Astrophysik Potsdam (AIP), Max-Planck-Institut für Astronomie (MPIA Heidelberg), Max-Planck-Institut für Astrophysik (MPA Garching), Max-Planck-Institut für Extraterrestrische Physik (MPE), National Astronomical Observatories of China, New Mexico State University, New York University, University of Notre Dame, Observatário Nacional / MCTI, The Ohio State University, Pennsylvania State University, Shanghai Astronomical Observatory, United Kingdom Participation Group, Universidad Nacional Autónoma de México, University of Arizona, University of Colorado Boulder, University of Oxford, University of Portsmouth, University of Utah, University of Virginia, University of Washington, University of Wisconsin, Vanderbilt University, and Yale University.

\section{References}

Adibekyan, V. Z., Sousa, S. G., Santos, N. C., et al. 2012, A\&A, 545, A32 Arenou, F., Luri, X., Babusiaux, C., et al. 2018, A\&A, 616, A17

Beers, T. C., Drilling, J. S., Rossi, S., et al. 2002, AJ, 124, 931

Bell, E. F., Zucker, D. B., Belokurov, V., et al. 2008, ApJ, 680, 295

Belokurov, V., Erkal, D., Evans, N. W., Koposov, S. E., \& Deason, A. J. 2018 MNRAS, 478, 611

Bensby, T., Alves-Brito, A., Oey, M. S., Yong, D., \& Meléndez, J. 2011, ApJ, 735, L46

Benson, A. J., Lacey, C. G., Frenk, C. S., Baugh, C. M., \& Cole, S. 2004, MNRAS, 351, 1215

Bonaca, A., Conroy, C., Wetzel, A., Hopkins, P. F., \& Kereš, D. 2017, ApJ, 845 101

Bovy, J., Rix, H.-W., Liu, C., et al. 2012, ApJ, 753, 148

Bovy, J., Rix, H.-W., Schlafly, E. F., et al. 2016, ApJ, 823, 30

Brown, W. R., Beers, T. C., Wilhelm, R., et al. 2008, AJ, 135, 564

Bullock, J. S., \& Johnston, K. V. 2004, in Satellites and Tidal Streams, eds.

F. Prada, D. Martinez Delgado, \& T. J. Mahoney, ASP Conf. Ser., 327, 80

Carollo, D., Beers, T. C., Lee, Y. S., et al. 2007, Nature, 450, 1020

Carollo, D., Beers, T. C., Chiba, M., et al. 2010, ApJ, 712, 692

Chen, B., Stoughton, C., Smith, J. A., et al. 2001, ApJ, 553, 184

Cheng, J. Y., Rockosi, C. M., Morrison, H. L., et al. 2012, ApJ, 752, 51

Chiba, M., \& Beers, T. C. 2000, AJ, 119, 2843

Cole, S. 1991, ApJ, 367, 45

De Lucia, G., \& Helmi, A. 2008, MNRAS, 391, 14

Eggen, O. J., Lynden-Bell, D., \& Sandage, A. R. 1962, ApJ, 136, 748

Fattahi, A., Belokurov, V., Deason, A. J., et al. 2019, MNRAS, 484, 4471

Fernández-Alvar, E., Tissera, P. B., Carigi, L., et al. 2019a, MNRAS, 485, 1745

Fernández-Alvar, E., Fernández-Trincado, J. G., Moreno, E., et al. 2019b, MNRAS, 487, 1462

Font, A. S., Navarro, J. F., Stadel, J., \& Quinn, T. 2001, ApJ, 563, L1

Font, A. S., Johnston, K. V., Bullock, J. S., \& Robertson, B. E. 2006, ApJ, 646, 886

Font, A. S., McCarthy, I. G., Crain, R. A., et al. 2011, MNRAS, 416, 2802

Forbes, D. A., \& Bridges, T. 2010, MNRAS, 404, 1203

Gaia Collaboration (Prusti, T., et al.) 2016, A\&A, 595, A1

Gaia Collaboration (Clementini, G., et al.) 2017, A\&A, 605, A79

Gaia Collaboration (Brown, A. G. A., et al.) 2018a, A\&A, 616, A1

Gaia Collaboration (Babusiaux, C., et al.) 2018b, A\&A, 616, A10

Gallart, C., Bernard, E. J., Brook, C. B., et al. 2019, Nat. Astron., 407

Gómez, F. A., Helmi, A., Brown, A. G. A., \& Li, Y.-S. 2010, MNRAS, 408, 935

Guiglion, G., Recio-Blanco, A., de Laverny, P., et al. 2015, A\&A, 583, A91

Hawkins, K., Jofré, P., Masseron, T., \& Gilmore, G. 2015, MNRAS, 453, 758

Hayes, C. R., Majewski, S. R., Shetrone, M., et al. 2018, ApJ, 852, 49

Haywood, M. 2008, MNRAS, 388, 1175

Haywood, M., Di Matteo, P., Lehnert, M. D., Katz, D., \& Gómez, A. 2013, A\&A, 560, A109

Haywood, M., Di Matteo, P., Snaith, O., \& Lehnert, M. D. 2015, A\&A, 579, A5

Haywood, M., Di Matteo, P., Lehnert, M. D., et al. 2018, ApJ, 863, 113
Helmi, A., \& de Zeeuw, P. T. 2000, MNRAS, 319, 657

Helmi, A., White, S. D. M., de Zeeuw, P. T., \& Zhao, H. 1999, Nature, 402, 53

Helmi, A., Babusiaux, C., Koppelman, H. H., et al. 2018, Nature, 563, 85

House, E. L., Brook, C. B., Gibson, B. K., et al. 2011, MNRAS, 415, 2652

Ibata, R. A., McConnachie, A., Cuillandre, J.-C., et al. 2017, ApJ, 848, 129

Ibata, R. A., Malhan, K., Martin, N. F., \& Starkenburg, E. 2018, ApJ, 865, 85

Iorio, G., \& Belokurov, V. 2019, MNRAS, 482, 3868

Jackson-Jones, R., Jofré, P., Hawkins, K., et al. 2014, A\&A, 571, L5

Jean-Baptiste, I., Di Matteo, P., Haywood, M., et al. 2017, A\&A, 604, A106

Johnson, D. R. H., \& Soderblom, D. R. 1987, AJ, 93, 864

Johnston, K. V., Bullock, J. S., Sharma, S., et al. 2008, ApJ, 689, 936

Kazantzidis, S., Bullock, J. S., Zentner, A. R., Kravtsov, A. V., \& Moustakas,

L. A. 2008, ApJ, 688, 254

Kepley, A. A., Morrison, H. L., Helmi, A., et al. 2007, AJ, 134, 1579

Koppelman, H., Helmi, A., \& Veljanoski, J. 2018, ApJ, 860, L11

Kordopatis, G., Gilmore, G., Wyse, R. F. G., et al. 2013, MNRAS, 436, 3231

Kruijssen, J. M. D., Pfeffer, J. L., Reina-Campos, M., Crain, R. A., \& Bastian,

N. 2019, MNRAS, 486, 3180

Leaman, R., VandenBerg, D. A., \& Mendel, J. T. 2013, MNRAS, 436, 122

Lee, Y. S., Beers, T. C., An, D., et al. 2011, ApJ, 738, 187

Lehnert, M. D., Di Matteo, P., Haywood, M., \& Snaith, O. N. 2014, ApJ, 789,

L30

Li, C., \& Zhao, G. 2017, ApJ, 850, 25

Lindegren, L., Hernández, J., Bombrun, A., et al. 2018, A\&A, 616, A2

Mackereth, J. T., Schiavon, R. P., Pfeffer, J., et al. 2019, MNRAS, 482, 3426

Majewski, S. R., Munn, J. A., \& Hawley, S. L. 1996, ApJ, 459, L73

Majewski, S. R., Schiavon, R. P., Frinchaboy, P. M., et al. 2017, AJ, 154, 94

Malhan, K., Ibata, R. A., \& Martin, N. F. 2018, MNRAS, 481, 3442

McCarthy, I. G., Font, A. S., Crain, R. A., et al. 2012, MNRAS, 420, 2245

Minchev, I., Chiappini, C., Martig, M., et al. 2014, ApJ, 781, L20

Morrison, H. L., Flynn, C., \& Freeman, K. C. 1990, AJ, 100, 1191

Moster, B. P., Macciò, A. V., Somerville, R. S., Johansson, P. H., \& Naab, T. 2010, MNRAS, 403, 1009

Myeong, G. C., Evans, N. W., Belokurov, V., Sanders, J. L., \& Koposov, S. E. 2018, ApJ, 863, L28

Navarro, J. F., Abadi, M. G., Venn, K. A., Freeman, K. C., \& Anguiano, B. 2011, MNRAS, 412, 1203

Nissen, P. E., \& Schuster, W. J. 2010, A\&A, 511, L10

Nissen, P. E., \& Schuster, W. J. 2011, A\&A, 530, A15

Norris, J., Bessell, M. S., \& Pickles, A. J. 1985, ApJ, 58, 463

Pillepich, A., Madau, P., \& Mayer, L. 2015, ApJ, 799, 184

Price-Whelan, A. M., \& Bonaca, A. 2018, ApJ, 863, L20

Purcell, C. W., Bullock, J. S., \& Kazantzidis, S. 2010, MNRAS, 404, 1711

Qu, Y., Di Matteo, P., Lehnert, M., van Driel, W., \& Jog, C. J. 2010, A\&A, 515, A11

Qu, Y., Di Matteo, P., Lehnert, M. D., \& van Driel, W. 2011a, A\&A, 530, A10

Qu, Y., Di Matteo, P., Lehnert, M. D., van Driel, W., \& Jog, C. J. 2011b, A\&A, 535, A5

Quinn, P. J., Hernquist, L., \& Fullagar, D. P. 1993, ApJ, 403, 74

Ramírez, I., Meléndez, J., \& Chanamé, J. 2012, ApJ, 757, 164

Reddy, B. E., \& Lambert, D. L. 2008, MNRAS, 391, 95

Reid, M. J., Menten, K. M., Brunthaler, A., et al. 2014, ApJ, 783, 130

Sahlholdt, C. L., Casagrande, L., \& Feltzing, S. 2019, ApJ, 881, L10

Schönrich, R., Binney, J., \& Dehnen, W. 2010, MNRAS, 403, 1829

Schuster, W. J., Moreno, E., Nissen, P. E., \& Pichardo, B. 2012, A\&A, 538, A21

Searle, L., \& Zinn, R. 1978, ApJ, 225, 357

Sestito, F., Longeard, N., Martin, N. F., et al. 2019, MNRAS, 484, 2166

Simion, I. T., Belokurov, V., \& Koposov, S. E. 2019, MNRAS, 482, 921

Snaith, O., Haywood, M., Di Matteo, P., et al. 2015, A\&A, 578, A87

Snaith, O. N., Haywood, M., Di Matteo, P., et al. 2014, ApJ, 781, L31

Sommer-Larsen, J., \& Zhen, C. 1990, MNRAS, 242, 10

Twarog, B. A., \& Anthony-Twarog, B. J. 1994, AJ, 107, 1371

Twarog, B. A., \& Anthony-Twarog, B. J. 1996, AJ, 111, 220

Velazquez, H., \& White, S. D. M. 1999, MNRAS, 304, 254

Venn, K. A., Irwin, M., Shetrone, M. D., et al. 2004, AJ, 128, 1177

Villalobos, Á., \& Helmi, A. 2008, MNRAS, 391, 1806

Villalobos, Á., \& Helmi, A. 2009, MNRAS, 399, 166

Walker, I. R., Mihos, J. C., \& Hernquist, L. 1996, ApJ, 460, 121

White, S. D. M., \& Frenk, C. S. 1991, ApJ, 379, 52

Xue, X.-X., Rix, H.-W., Yanny, B., et al. 2011, ApJ, 738, 79

Zinn, R. 1993, in The Globular Cluster-Galaxy Connection, eds. G. H. Smith, \& J. P. Brodie, ASP Conf. Ser., 48, 38

Zinn, R. 1996, in Formation of the Galactic Halo... Inside and Out, eds. H. L

Morrison, \& A. Sarajedini, ASP Conf. Ser., 92, 211

Zolotov, A., Willman, B., Brooks, A. M., et al. 2010, ApJ, 721, 738 


\section{Appendix A: Velocity uncertainties}
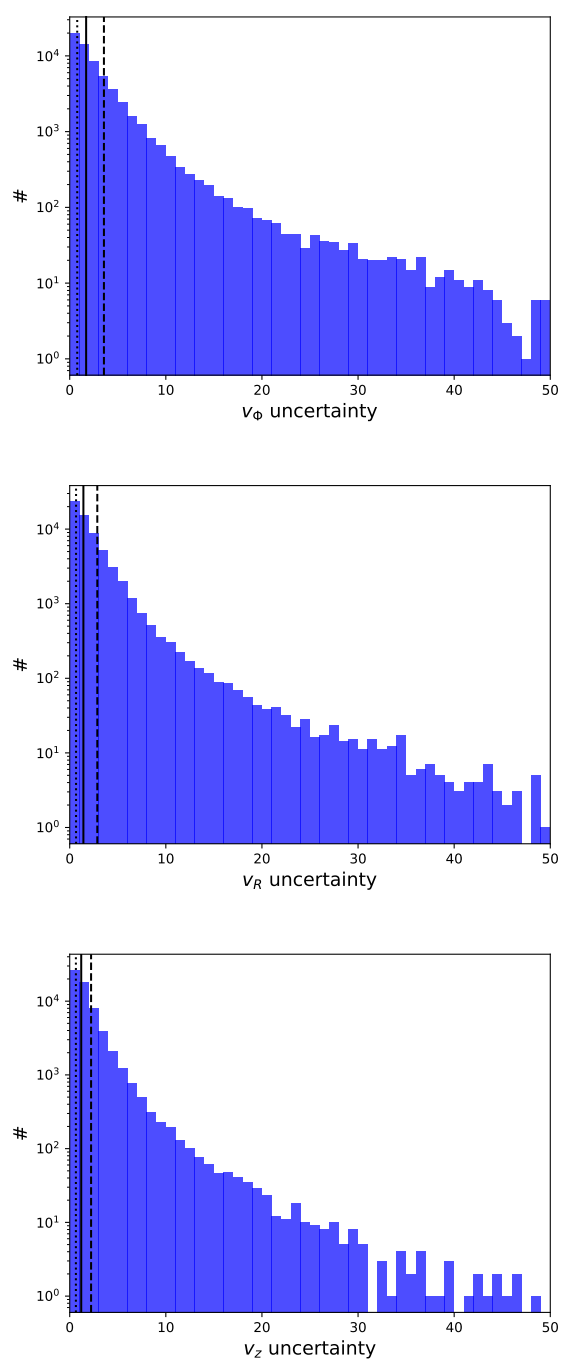

Fig. A.1. Distribution of velocity uncertainties on $v_{\Phi}$ (top panel), $v_{r}$ (middle panel) and $v_{z}$ (bottom panel), due to propagation of individual errors on observables. In each panel, the dottted, solid and dashed lines indicate, respectively, the 25th, 50th (median) and 75th percentile of the distribution. All uncertainties are given in units of $\mathrm{km} \mathrm{s}^{-1}$.

In this section, we firstly discuss the uncertainties in the velocities of stars in the sample, due to the propagation of the individual uncertainties in the observables (parallaxes, proper motions, and radial velocities). Then, we discuss how these uncertainties impact the velocities (and velocity dispersions)-abundances relation presented in Sect. 3.1. We anticipate the conclusions of this section, by confirming that the statistical uncertainties estimated by the bootstrapping technique adopted in Sect. 3.1 are always the dominant source of uncertainty, when uncertainties are significant.

To estimate the uncertainties on the azimuthal, radial and vertical velocities $v_{\Phi}, v_{R}, v_{z}$, of each star, due to the propagation of the individual uncertainties on its parallax, proper motion and radial velocity, we have assumed gaussian distributions of these errors, and generated 100 random realisations of these parameters per source. For each realisation, we then make a transformation from the space of observables $\left(\alpha, \delta, \pi, \mu_{\alpha}{ }^{*}, \mu_{\delta}\right)$ to the Galactocentric rest-frame (see Johnson \& Soderblom 1987; Kepley et al. 2007), and calculate the corresponding values of $v_{\Phi}, v_{R}, v_{z}$. The resulting uncertainties on $v_{\Phi}, v_{R}, v_{z}$ are then finally estimated as the standard deviations of their values, over the 100 realisations. Their distributions are shown in Fig. A.1, and probe that for most of the stars uncertainties are low: the 75th percentile of the distribution is indeed equal to $3.56 \mathrm{~km} \mathrm{~s}^{-1}$, $2.87 \mathrm{~km} \mathrm{~s}^{-1}$ and $2.22 \mathrm{~km} \mathrm{~s}^{-1}$, for $v_{\Phi}, v_{R}$ and $v_{z}$, respectively, whereas the corresponding medians are $1.70 \mathrm{~km} \mathrm{~s}^{-1}, 1.42 \mathrm{~km} \mathrm{~s}^{-1}$, and $1.20 \hat{\mathrm{A}} \mathrm{km} \mathrm{s}^{-1}$.

Uncertainties on $v_{\Phi}, v_{R}, v_{z}$ increase with the distance of stars in the sample from the Sun, as expected (see Fig. A.2, first column). Stars with halo kinematics $\left(v_{\Phi} \sim 0 \mathrm{~km} \mathrm{~s}^{-1}\right.$, high $v_{R}$ and $v_{z}$ ) also have, on average, larger uncertainties than stars with disc-like kinematics (see Fig. A.2, second column), and this finding can be explained because the fraction of halo stars increases with the distance from the Sun. Despite the larger uncertainties, the typical (median) uncertainty of stars with null $v_{\Phi},\left|v_{r}\right| \geq 150 \mathrm{~km} \mathrm{~s}^{-1}$ and $\left|v_{r}\right| \geq 100 \mathrm{~km} \mathrm{~s}^{-1}$ is equal to, or below, $15 \mathrm{~km} \mathrm{~s}^{-1}$.

Finally, because stars with halo-like kinematics have, on average, larger velocity uncertainties than stars with disc-like kinematics, a trend exists between the $v_{\Phi}, v_{R}$ and $v_{z}$ uncertainties and chemical abundances, with the uncertainties decreasing with $[\mathrm{Fe} / \mathrm{H}]$, and increasing with $[\mathrm{Mg} / \mathrm{Fe}]$ (see Fig. A.2, third and fourth columns).

The uncertainties on the velocity dispersions-abundance relations discussed in Sect. 3.1 have been estimated by making use of the 100 random realisations described before. For each realisation, we computed the corresponding velocity dispersionabundance relations (see Fig. A.3, middle and bottom plots, top panels), and estimated the corresponding standard deviations (see Fig. A.3, middle and bottom plots, bottom panels). As shown in this Figure, the uncertainties on the velocity dispersion-abundance relations are always very small, and most of the time below $5 \mathrm{~km} \mathrm{~s}^{-1}$ (for the velocity dispersions-[Fe/H] relation), and below $1.5 \mathrm{~A} \mathrm{~km} \mathrm{~s}^{-1}$ (for the velocity dispersions$[\mathrm{Mg} / \mathrm{Fe}]$ relation). For completeness, and for comparison with Fig. A.2, we also show the dependency of the uncertainties in the velocity dispersions on the distance $D$ from the Sun in Fig. A.3. The comparison of the uncertainties on the velocity dispersions, due to the propagation of individual errors on observables and the uncertainties calculated with the bootstrapping technique (see Sect. 3.1) is shown in Fig. A.4, and demonstrates that the statistical uncertainties calculated by bootstrapping are always larger than (or equal to) those down to individual errors. In particular, when uncertainties in the sample are significant (above $2-3 \mathrm{~km} \mathrm{~s}^{-1}$ ), those calculated with the bootstrapping technique are always the dominant. As a consequence, the errors reported in Figs. 3 and 4, and estimated by bootstrapping the sample, constitute the dominant uncertainty among stars in our sample. 
P. Di Matteo et al.: Composition of the stellar halo and age-dating the last significant merger
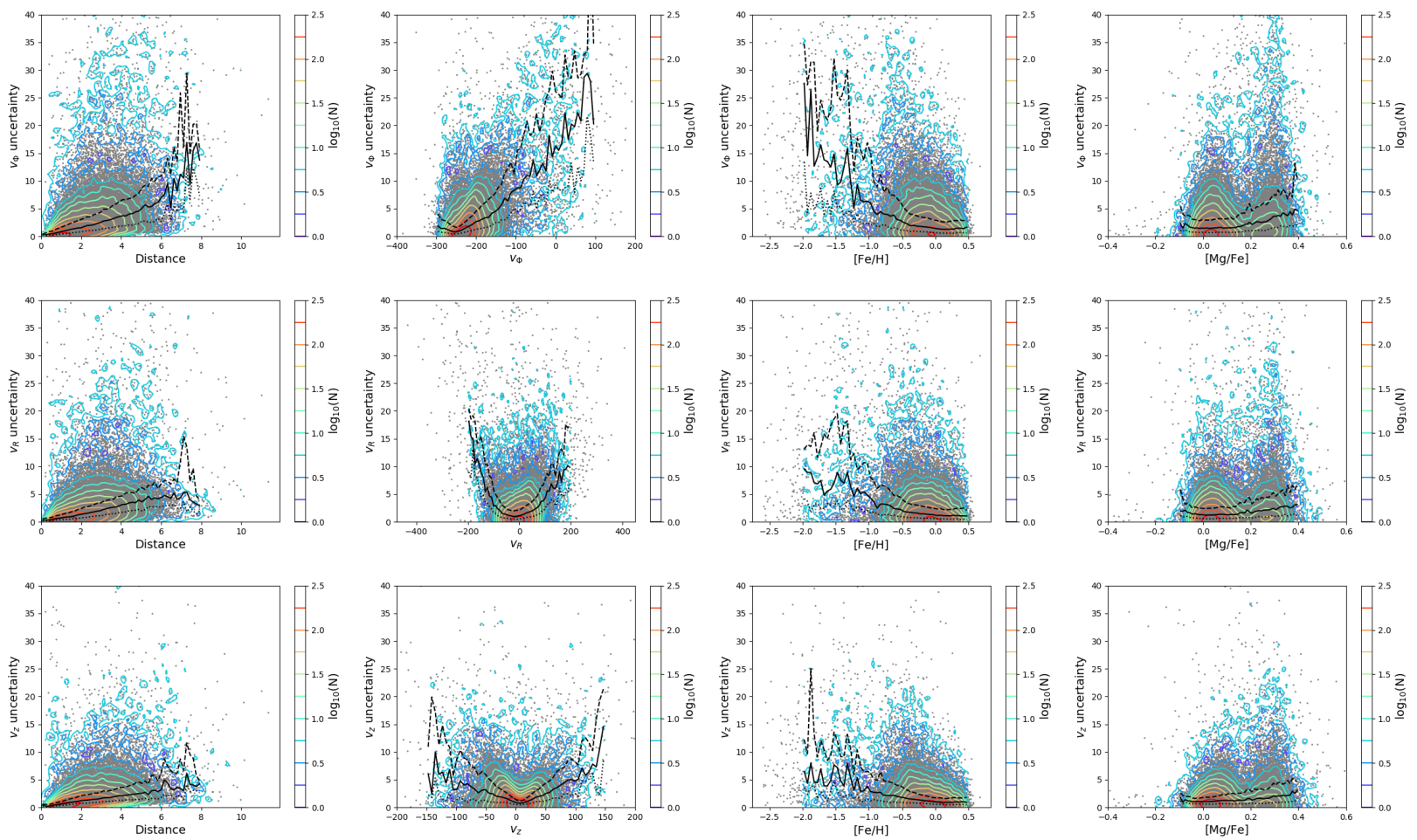

Fig. A.2. First column: uncertainties in $v_{\Phi}$ (first row), $v_{R}$ (second row) and $v_{z}$ (third row) of stars as a function of their distance $D$ from the Sun. Distances are given in kpc. Second column: uncertainties in $v_{\Phi}$ (first row), $v_{R}$ (second row) and $v_{z}$ (third row) of stars as a function of corresponding velocity (given in $\mathrm{km} \mathrm{s}^{-1}$ ). Third column: uncertainties in $v_{\Phi}$ (first row), $v_{R}$ (second row) and $v_{z}$ (third row) of stars as a function of [Fe/H]. Fourth column: Uncertainties in $v_{\Phi}$ (first row), $v_{R}$ (second row) and $v_{z}$ (third row) of stars as a function of $[\mathrm{Mg} / \mathrm{Fe}]$. In all panels: individual uncertainties are shown with grey points; their density distributions, in logarithmic scale, are indicated by coloured contours, whose corresponding values are reported in the error bars; the dotted, solid and dashed black lines show, respectively, the 25th, 50th (median) and 75th percentile of the distribution of uncertainties, as a function of the value reported on the $x$-axis. All velocity uncertainties are given in units of $\mathrm{km} \mathrm{s}^{-1}$. 

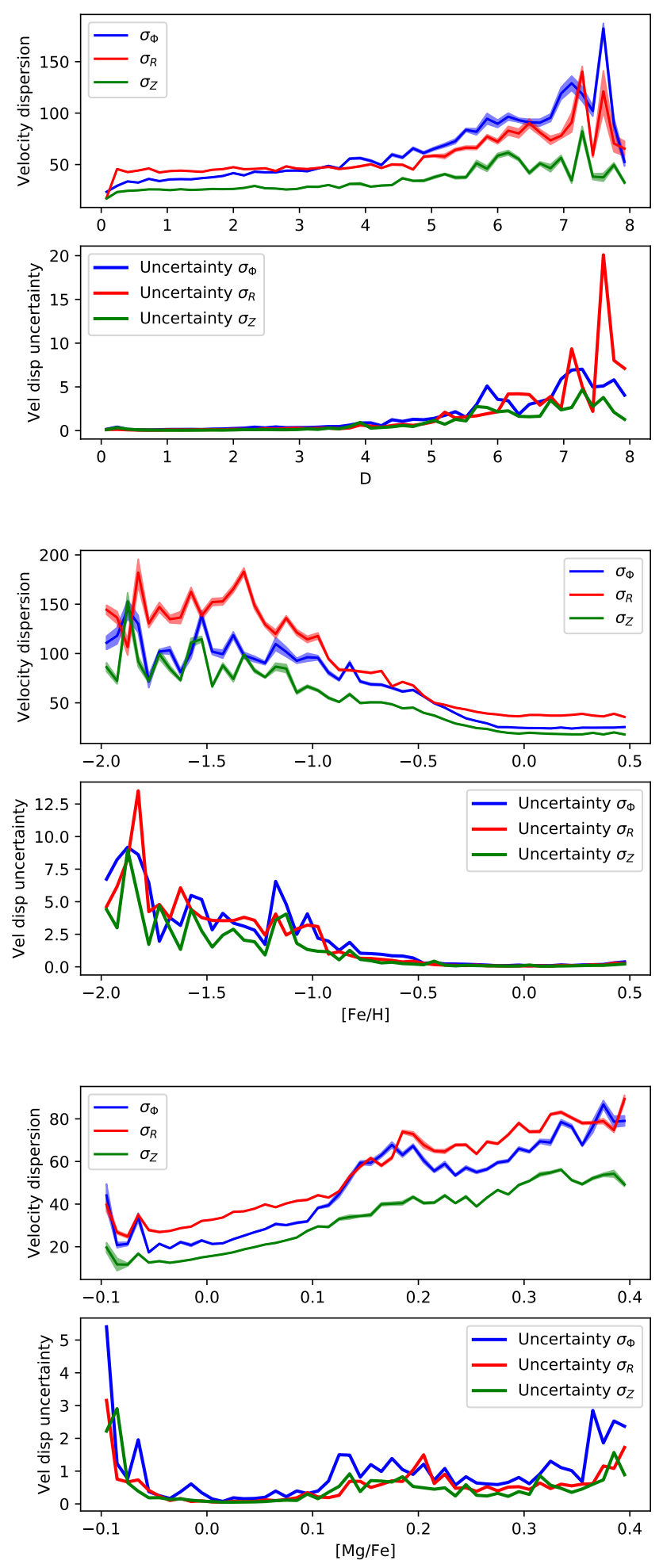

Fig. A.3. Top figure: velocity dispersion (top panel) and velocity dispersion uncertainty (bottom panel) as a function of distance $D$ from the Sun. Blue, red and green curves correspond to the velocity dispersions in the aziumthal, radial and vertical directions, as indicated in the legend. Bottom panel: solid lines indicate the mean relation, and the colour-shaded areas indicate the $1 \sigma$ uncertainty, estimated through 100 random realisations, from the uncertainties on the observables (see text). These uncertainties are then reported in the bottom panel. Middle and bottom figures: as above, but now velocity dispersions and corresponding uncertainties are shown as a function of $[\mathrm{Fe} / \mathrm{H}]$ and $[\mathrm{Mg} / \mathrm{Fe}]$, respectively. In all plots, velocities are in units of $\mathrm{km} \mathrm{s}^{-1}$.
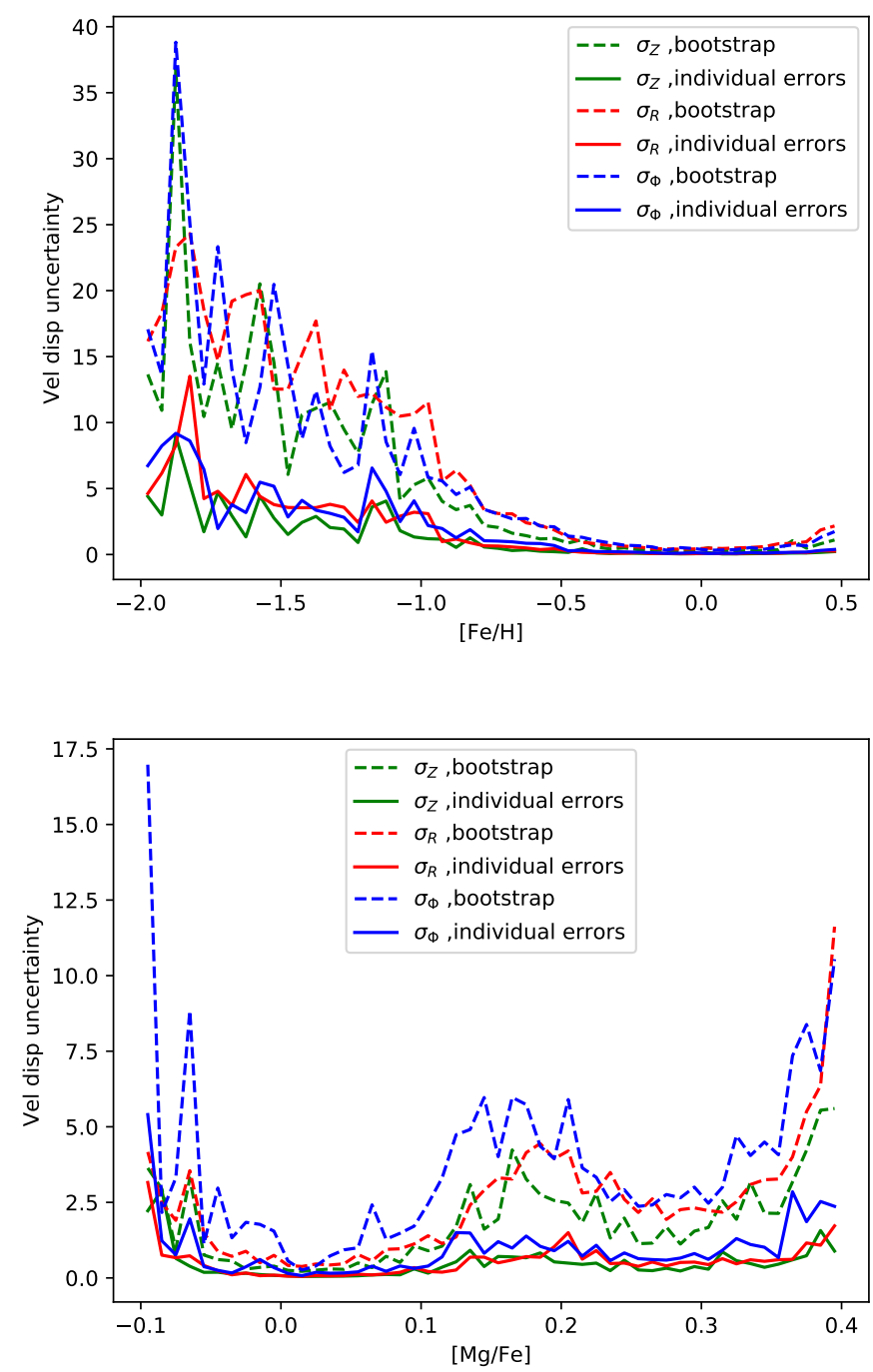

Fig. A.4. Comparison between uncertainties on velocity dispersions, due to propagation of uncertainties on individual observables (solid lines, see Appendix A for details) and those estimated by bootstrapping technique (dashed lines, see Sect. 3.1 for details). The uncertainties are shown as a function of $[\mathrm{Fe} / \mathrm{H}]$ (top panel) and as a function of $[\mathrm{Mg} / \mathrm{Fe}]$ (bottom panel).

\section{Appendix B: Number of stars in the mean chemo-kinematic relations}

In Fig. B.1, we present the number distribution of stars, as a function of their $[\mathrm{Mg} / \mathrm{Fe}](/[\mathrm{Fe} / \mathrm{H}])$ ratio, for different $[\mathrm{Fe} / \mathrm{H}]$ and $[\mathrm{Mg} / \mathrm{Fe}]$ intervals. The adopted values for the $[\mathrm{Fe} / \mathrm{H}]$ and $[\mathrm{Mg} / \mathrm{Fe}]$ intervals are the same as those already used in Sect. 3.1 and Figs. 3-5, those are ten metallicity intervals, ranging from $[\mathrm{Fe} / \mathrm{H}]=-2.1$ up to $[\mathrm{Fe} / \mathrm{H}]=0.25$ (see Fig. B.1, top panel), and six $[\mathrm{Mg} / \mathrm{Fe}]$ intervals, ranging from $[\mathrm{Mg} / \mathrm{Fe}]=-0.1$ up to $[\mathrm{Mg} / \mathrm{Fe}]=0.4$ (see Fig. B.1, bottom panel). The width of the intervals is the same as adopted in Sect. 3.1. We point out that in Fig. B.1, in both panels, we used a number of bins along the $x$-axis greater than those used in Figs. 3-5, to make it clear where the dip in the $[\mathrm{Mg} / \mathrm{Fe}]-[\mathrm{Fe} / \mathrm{H}]$ sequence is, and how it changes with $[\mathrm{Mg} / \mathrm{Fe}]$ for different $[\mathrm{Fe} / \mathrm{H}]$ intervals (see, for example, in the top panel, the values corresponding to $[\mathrm{Mg} / \mathrm{Fe}]=[0.24,0.27,0.21,0.18,0.18,0.18,0.12]$ for $[\mathrm{Fe} / \mathrm{H}]=[-1.5,-1.25,-1.0,-0.75,-0.5,-0.25,0.0])$. 
P. Di Matteo et al.: Composition of the stellar halo and age-dating the last significant merger
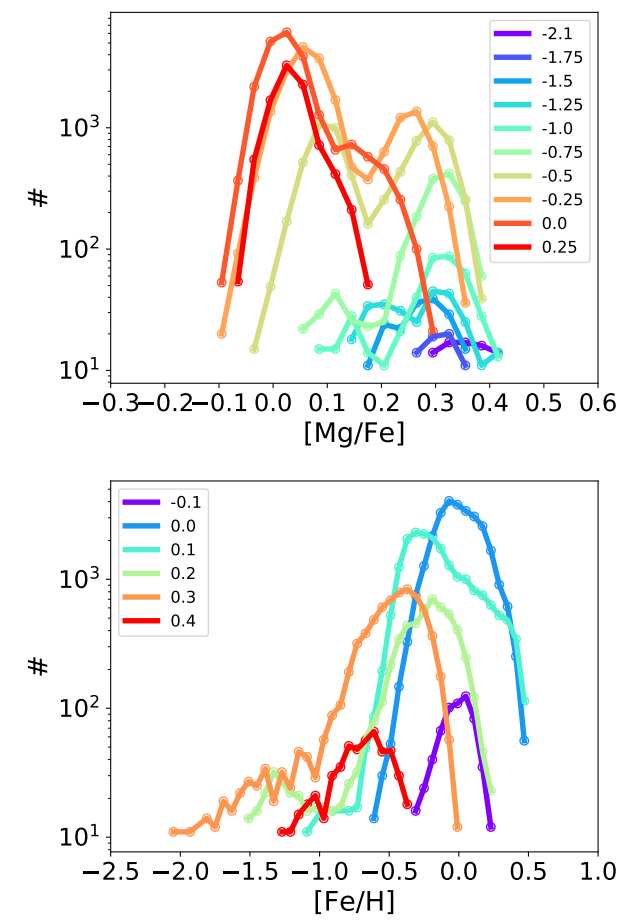

Fig. B.1. Top panel: number of stars, as a function of their $[\mathrm{Mg} / \mathrm{Fe}]$ ratio. Relations are given for bins in $[\mathrm{Fe} / \mathrm{H}]$, as indicated in the legend. Bottom panel: number of stars, as a function of their $[\mathrm{Fe} / \mathrm{H}]$ ratio. Relations are given for bins in $[\mathrm{Mg} / \mathrm{Fe}]$, as indicated in the legend. 